\title{
Die Situation der ausländischen Jugendlichen auf der Sekundarstufe II in der Schweizer Schule - Integration oder Benachteiligung?
}

\section{Romano Müller}

Gemäss der Theorie des Humankapitals müssen die Schulen alles daran setzen, die Bildungsressourcen ihrer Schülerinnen und Schüler optimal zu nutzen. Der Vergleich ausgewählter Nationengruppen auf der Sekundarstufe II mit autochthonen Schweizergruppen weist aber für die Schweiz nach, dass für ausländische Jugendliche die gesamte Bildungsbeteiligung niedrig ist. Besonders erschwert ist für die ausländischen Jugendlichen der Zugang zu den höheren Ausbildungsgängen der Mittelschulen, aber auch den anspruchsvolleren Berufslehren. Hier allerdings lässt sich ein Kompensationseffekt feststellen, der darin besteht, dass ein relativ hoher Anteil von Ausländern in den Berufslehren ebenso anspruchsvolle Berufe wählen wie die Schweizerinnen und Schweizer. Der Autor kritisiert den Mechanismus im schweizerischen Bildungssystem, durch den monolinguale Schüler selbst bei schlechteren schulischen Voraussetzungen dem bilingualen Ausländer vorgezogen wird als Präferentialismus, der die Interessen der autochthonen Gruppe schützt, längerfristig aber die sozialen und ökonomischen Interessen des Staates unterläuft.

\section{Die Probleme}

Im vorliegenden Artikel wird die Auffassung vertreten, dass die Angleichung des durchschnittlichen schulischen Erfolgs der ausländischen Schülerinnen und Schüler und Jugendlichen an jenen der einheimischen Schülerinnen und Schüler ein wesentlicher Gradmesser für die Bereitschaft bzw. das Potential einer majoritären Gesellschaft ist, ausländische Schülerinnen und Schüler und Jugendliche zu integrieren. Für die Gruppe der Jugendlichen werden (1) der (quantitative) Umfang der Bildungsbeteiligung und (2) das Ausbildungsniveau auf der Sekundarstufe II als objektiv messbare Kriterien gewählt. Mit dieser operationalen Festlegung des Begriffs Integration weiche ich bewusst von allgemeinen Formulierungen zur Integration von Migrantinnen und Migranten ab, wie sie oft in Lehrplänen und Weisungen zu finden sind. Dort ist etwa von «kulturelle[r] und 
sprachliche[r] Gleichwertigkeit und Gleichberechtigung» (Erziehungsdirektion des Kt. Bern, 1993) die Rede, ohne dass dabei bedacht würde, dass strukturelle Gegebenheiten - wie etwa die ausgeprägte Monolingualität der Schule - solchen Forderungen nach Gleichheit zuwiderlaufen. Es liegt auf der Hand, dass mit dieser Definition nicht das gesamte Spektrum anderer möglicher Integrationsparameter abgedeckt wird. So sind beispielsweise rechtliche, soziale und arbeitsweltbezogene Aspekte der Integration durch diese Definition nicht impliziert. Man muss aber bedenken, dass mit der Bildungsbeteiligung verschiedene Bereiche des privaten und gesellschaftlichen Wohlergehens in Zusammenhang stehen.

1. Gesichtspunkt des Humankapitals: Es besteht unzweifelhaft ein sehr enger Zusammenhang zwischen schulischem Erfolg und ökonomischen Parametern wie Stellung in der Arbeitswelt, Einkommen, Nutzung von Bildungsangeboten, Faktoren also, welche gemeinhin unter dem Begriff der Bildungsrendite zusammengefasst werden. Unter einer personalen Betrachtungsweise erfahren statistisch gesehen jene ausländischen Menschen ökonomische und gesellschaftliche Nachteile, die, obwohl sie über die intellektuellen Voraussetzungen verfügten, zu einer geringeren Ausbildung als ihre autochthone Vergleichsgruppe gelangen. Vom gesellschaftlichen Gesichtspunkt her besteht ein starker - wenn auch nicht in allen Staaten gleich ausgeprägter - Zusammenhang zwischen Bildungsbeteiligung einerseits und ökonomischen Faktoren wie Erwerbstätigkeit, Erwerbseinkommen, Fiskalität oder allgemeiner: sozialer Bildungsrendite andererseits (BfS, 1998, 1999). Vereinfacht gesagt heisst dies: Moderne Gesellschaften ziehen soziale und ökonomische Vorteile aus (1) dem Grad der Bildungsbeteiligung und (2) aus dem Ausbildungsgrad ihrer Bevölkerung. Ein solcher, der Theorie des Humankapitals verpflichteter Ansatz (neuerdings: Alsalam \& Conley, 1995; Grin, 1994; OECD, 1998; Psacharopoulos, 1987, 1994; Wolter \& Weber, 1998, 1999a, 1999b), betrachtet die Bildungsbeteiligung somit unter der Fragestellung, inwiefern ein Bildungssystem Vor- oder Nachteile für die Individuen und die Gesellschaft bringt. Darüber hinaus bestehen selbstverständlich oft schwer zu quantifizierende Zusammenhänge zwischen dem Bildungsgrad einer Bevölkerungsgruppe und Faktoren wie etwa körperlicher und psychischer Gesundheit (zusammenfassend BfS, 1998, S. 48ff.), Suchtmittelkonsum (Neuenschwander, 1997; Süss \& Neuenschwander, 1997), sozialer Auflehnung (Bolzmann \& Eckmann, 1997; Eckmann-Saillant \& Bolzman, 1994) oder gar Kriminalität. Wir errechneten beispielsweise einen hohen Zusammenhang von $\mathrm{r}$ zwischen 0.70 und 0.89 zwischen dem Kriminalitätsquotienten (Tatverdächtige 10 bis 17-jährige Jugendliche im Kanton Zürich) und dem Schulversagerquotienten gleichaltriger Schülerinnen und Schüler und Jugendlicher

\section{Gesichtspunkt der sozialen Wohlfahrt und wirtschaftlichen Entwicklung:}

Verwandt mit dieser bildungsökonomischen Betrachtungsweise ist die Frage nach dem Zusammenhang der Bildungsbeteiligung einerseits und dem Bezie- 
hungsgefüge zwischen rückläufiger Geburtenquote (Überalterung) und dem Mangel an qualifizierten Arbeitskräften andererseits. Für die Schweiz wird unter der Annahme eines mittleren Szenarios (TREND) des Bundesamtes für Statistik davon ausgegangen, dass sich der Alterslastquotient von heute ca. 4 Personen im Erwerbsalter auf 1 Person im Rentenalter bis zum Jahr 2020 auf 2.9 und bis 2035 auf ca. 2.2 reduzieren wird (BfS, 1996). Für Deutschland wäre eine jährliche zusätzliche Zuwanderung von 1/2 Mio. Menschen notwendig, um 2050 einen der heutigen Zahl ähnlichen Alterslastquotienten aufrechtzuerhalten (Klingst, 2000). Für die «Lösung» dieses Problems bieten sich zur Zeit fünf theoretische Möglichkeiten an, wobei mindestens die zwei letzteren mit der Integration von Ausländern in engem Zusammenhang stehen: (1) die Erhöhung des durchschnittlichen Rentenalters, (2) der Abbau der sozialen Wohlfahrt, (3) Anreize zur Erhöhung der Geburtenquote durch eine familienfreundliche Steuerund Sozialpolitik, (4) die Erhöhung der Zuwanderungszahl in den EU-Ländern und (5) die Steigerung des wirtschaftlichen Wachstums u.a. durch den Zuzug qualifizierten Personals aus Staaten ausserhalb des EU-Raums. Der Mangel an qualifizierten Personen kennzeichnet den Beginn einer brisanten Bildungskrise: Die hochindustrialisierten westlichen Länder vermögen heute ihren Bedarf an technologisch qualifizierten Arbeitskräften aus eigener Kraft nicht mehr zu dekken und sind deshalb dabei, Arbeitskräfte aus dem osteuropäischen und asiatischen Raum einzustellen. Die sonst einwanderungsrestriktiven USA haben die Einwanderungsquote für diese Berufsgruppen 1999 von 20'000 auf $115^{\prime} 000$ erhöht (Morice, 2000); die deutsche Regierung hat für das Jahr 2000 allein eine Zuwanderung von 20'000 «TechnologInnen» angekündigt, und die «Kommission für Zuwanderung» ${ }^{1}$ empfiehlt eine gesteuerte Einwanderung von 50 '000 Immigrantinnen und Immigranten pro Jahr. Es ist aber auch davon auszugehen, dass global tätige Unternehmen die Problemlösung durch Ausgliederung der Arbeit in Billiglohnländer suchen und dass die westeuropäischen Nationalstaaten somit dieses Anteils am Bruttosozialprodukt verlustig gehen werden.

3. Gesichtspunkt sozialer Spannungen: Die Politologin Saskia Sassen (1999, 2000) hat die Einwanderungspolitiken der westeuropäischen Länder als jene kritisiert, die grundsätzlich widersprüchlich verlaufen. Kurz zusammengefasst: Die globalisierenden Massnahmen von westlichen Unternehmen und Staaten lösten Wanderungsbewegungen aus, die dann durch restriktive Massnahmen - wie etwa jener des den osteuropäischen Staaten aufgezwungenen «Cordon sanitaire» - eingedämmt werden sollten. In der sich abzeichnenden Selektionspolitik von erwünschten vs. nicht erwünschten Ausländerinnen und Ausländern lässt sich eine hochgradige soziale Sprengkraft ausmachen: Arbeitsmobile, neu zugezogene qualifizierte Ausländerinnen und Ausländer mit hohen beruflichen Qualifikationen und sozialen und bildungsmässigen Ansprüchen für ihre Kinder, jedoch ohne präzise Sprachkenntnisse und kulturelle Erfahrungen in der Einwanderungsgesellschaft, treten in Konkurrenz (a) zu den autochthonen Gruppen des 
gleichen sozialen Status und (b) zu jener im Einwanderungsland geborenen (umgangs-)sprachlich und kulturell hoch integrierten, aber sozialschichtig oft niedrigeren Grossgruppe von Ausländerinnen und Ausländern, die man kurz als die «zweite Generation» bezeichnet. (c) Dabei bleibt jene Gruppe von (unerwünschten) Migranten, die aus kriegsversehrten Ländern oder aus wirtschaftlicher Not ihr Land verlassen haben, noch unberücksichtigt.

Der skizzierte Problemrahmen lässt bereits Folgendes erkennen: (1) Die Probleme können nicht (allein) durch Erziehung gelöst werden, vielmehr ist deren Behebung die Aufgabe aller staatlichen und auch überstaatlichen Institutionen. (2) Der Pädagogik erwächst die Aufgabe, der mehrsprachigen Vielfalt vor allem mittels schulisch-struktureller und weniger mit didaktisch-unterrichtlichen Massnahmen Rechnung zu tragen. (3) Die Steigerung der Bildungsbeteiligung der ausländischen Schülerinnen und Schüler ist eine zentrale Aufgaben der multikulturellen Schule, sofern sie ihrem Integrationsauftrag gerecht werden will.

Es wird hier die Auffassung vertreten, dass das grundlegende schulisch-strukturelle Problem der Integration ausländischer Schülerinnen und Schüler im ausgeprägten Monolingualismus und dem damit verbundenen Selektionseffekt besteht. Damit verbinde ich die Behauptung, dass ohne die Reform der monolingualen Schule und deren Selektionsmechanismen fast alle unterrichtlichen Massnahmen der sogenannten interkulturellen Pädagogik in ihrer Wirkung gering bleiben werden.

\section{Der Grundmechanismus des Bildungsmisserfolgs: Der Monolingualismus als Voraussetzung von Schulerfolg}

Vorbemerkung: Ich werde an dieser Stelle bewusst nicht auf die Selektion auf der Primarstufe und im Übergang von der Primarstufe zur Sekundarstufe I eingehen, sondern mich auf die Situation an der nachobligatorischen Sekundarstufe II (Mittelschulen; Berufslehren, sog. Zwischenlösungen) beschränken. Die Sachverhalte für die obligatorische Schule wurden in der ganzen Breite an verschiedenen Stellen ausgeführt (Borkowsky, 1996; Doudin, 1998; Haeberlin \& Ekkhart, 2000; Hutmacher, 1995; Kronig, 1996, 1997; Kronig, Moser \& Rhyn, 1996, 1997, 1999; Lischer, 1997; Müller, 1993, 1996a, 1996b, 1997, 2000; Rüesch, 1998). Das Fazit ist eindeutig: Der schulische Misserfolg der ausländischen Migrantenkinder ist auch unter Kontrolle von Parametern wie soziale Herkunft; Intelligenz, Geschlecht, Dauer des Schulbesuchs und Schultypus im Vergleich zu den gleichaltrigen Schweizerinnen und Schweizer überwältigend hoch. Sowohl Rüesch (regressionsanalytisch; 1998; S. 290) wie auch Müller (varianzanalytisch: 1993, 1996a, S. 69; 1997, S. 235) kommen aufgrund unterschiedlicher Verfahren zum Schluss, dass beispielsweise bei vergleichbarem sozio-ökonomischem Hintergrund Migrantenkinder niedrigere schulsprachliche 
Leistungen erbringen als Schweizerinnen und Schweizer. Moser \& Rhyn (1999, S. 7f.) errechnen aufgrund einer Kontextanalyse den Einfluss der sozialen Herkunft je nach Klassenzusammensetzung auf geringe 3\%-5\% Unterschied bei der Lösung sprachlicher Aufgaben und auf lediglich 0.4\% bei mathematischen Aufgaben. Auch wenn die Faktoren der sozioökonomischen Zugehörigkeit oder des Geschlechts selbstverständlich wirksam sind, so lässt sich die Leistungsvarianz nicht auf diese reduzieren. Vielmehr dominiert der Parameter ethnolinguistische Zugehörigkeit die anderen «soziologischen» Parameter bezüglich der Wirkung auf den Schulerfolg (Müller, 1996a, 69ff.; Lischer, 1997, S. 12 zur Kontroverse).

Es ist wichtig, zu sehen, dass es sich bei den Schülerinnen und Schülern dieser Studien nicht einfach um neu zugezogene Kinder handelt; der überdurchschnittliche schulische Misserfolg betrifft auch die Kinder der zweiten Generation, also jene Kinder, welche die Umgangssprache der majoritären monolingualen Gesellschaft vollständig beherrschen, die Schweiz als ihre gewohnte alltägliche Umgebung kennen und dieses Land in der Regel als ihre Heimat und deren Umgangssprache als ihre Muttersprache bezeichnen (Müller, 1997). Es ist daher klar, dass die Erfolgs-Misserfolgsstatistiken der Sekundarstufe II in einem hohen Grade durch jene der vorherigen Schulstufen mit bedingt sind.

Monolingualer Habitus: Zentraler für die jetzige Diskussion ist der Umstand, dass es offenbar der monolinguale Habitus (Gogolin, 1994) der öffentlichen Schule ist, der den Misserfolg zumeist zweisprachiger Kinder wesentlich mit verursacht. Eine relativ grosse Gruppe von ausländischen Schülerinnen und Schüler wird aufgrund der Tatsache, dass die monolinguale Schule ihre Mehrsprachigkeit kaum oder dann schon eher als Störfaktor beachtet, gar nie bestimmte Ausbildungsgänge der Sekundarstufe II einschlagen können, obwohl diese Schülerinnen und Schüler unter einer nicht-(schul-)sprachlichen Betrachtungsweise dazu durchaus geeignet wären (Müller, 2000). Einem Teil der ausländischen Jugendlichen werden somit Bildungskarrieren vorenthalten, für die sie eigentlich «begabt» wären. Unter dem Gesichtspunkt der Bildungsökonomie geht deren Bildungspotential durch Unterforderung zum Schaden der Volkswirtschaft und der Gesellschaft teilweise verloren.

Dieser selektive Vorgang liegt selbstverständlich im Widerspruch zum ökonomischen Bedarf eines Landes, welches auf die optimale Nutzung des potentiell vorhandenen humanen Kapitals angewiesen ist (und dieses oft importieren muss!). Er steht auch im Widerspruch zur behaupteten Absicht der Integration und Nicht-Diskriminierung anderssprachiger Menschen durch die Gesetzgebung bzw. die Verfassung demokratischer Gesellschaften. Er gliedert sich aber umgekehrt fast lückenlos in die soziale Logik der Grenzziehung zwischen unterschiedlichen Gruppen ein, wie sie von der Intergruppentheorie des sozialen Verhaltens ausführlich beschrieben wird (etwa Giles, 1977; Giles \& Byrne, 1982; Giles, Bourhis \& Taylor, 1977; Tajfel, 1978, 1982; Turner, 1982; Turner \& Brown, 1978; Müller, 1997 im Überblick). Dabei versuchen die jeweils gegenei- 
nander konkurrierenden Gruppen ihre kulturellen, sozialen und ökonomischen Interessen durch Grenzziehungen entlang von bestimmten Merkmalen bzw. Kategorisierungen zu ziehen und definieren je nach Bedarf diese Gruppengrenzen als «hart» oder "weich». Da in demokratischen Gesellschaften Grenzziehungen entlang der Merkmale wie Hautfarbe, Geschlecht, Religion und Sprache verfassungsrechtlich untersagt sind, "besinnen» sich diese raffinierterer kompetitiver Strategien, die sie des Vorwurfs des Verfassungsbruchs entheben. So ist die schulische Selektion - insbesondere jene mittels des sprachlichen Standards (Schulsprache) - eines der probaten Mittel majoritärer Gesellschaftsgruppen, die sozialen und ökonomischen Wettbewerbschancen anderssprachiger Ausländerinnen und Ausländer zu beschneiden oder genauer: zu regulieren. Indem man den Standard der majoritären Gesellschaft zum einheitlichen Selektionskriterium aller Schülerinnen und Schüler erhebt, entgeht man gleichzeitig dem verfassungsrechtlichen Vorwurf der Diskriminierung: Alle müssen ja das gleiche Selektionskriterium bestehen, um schulisch erfolgreich zu sein. Dieser Strategie entspricht die Praxis aller westeuropäischen Nationen. Es ist dies ein wesentlicher Grund dafür, warum diese Staaten am sprachlichen Territorialitätsprinzip festhalten. In einem gewissen (bewusst strapazierten) Sinne kann man sagen: Wer den (schriftlichen) Standard der jeweiligen Nationalsprache nicht durchschnittlich beherrscht, gilt als nicht oder noch nicht ganz integriert und kann darum auch nicht Anspruch auf den vollen Umfang der Güter einer Nation haben ${ }^{2}$.

Hier finden wir auch die Erklärung dafür, weshalb es zum jetzigen Zeitpunkt schulisch gesehen völlig unerheblich ist, ob ein ausländischer Schüler über zwei «Muttersprachen» verfügt. Unerheblich ist es deshalb auch, ob nachgewiesen werden kann, dass der quasi perfekte normative Beherrschungsgrad des sprachlichen Standards für die wenigsten beruflichen Karrieren von Belang ist ${ }^{3}$. Es sind daher auch nicht linguistische Argumente, welche den Wandel des monolingualen Habitus der Schule bewirken werden, sondern - wenn schon - das utilitaristische Interesse der majoritären Gesellschaft.

Wenn im Folgenden die Bildungsbeteiligung ausländischer Jugendlicher der Sekundarstufe II zur Diskussion steht, dann hat die schulisch-sprachliche Selektion ihre Wirkung bereits entfaltet und die Bildungswege bzw. deren Auswahlmöglichkeit sind weitgehend festgelegt. Wir werden aber sehen, dass die Benachteiligung ausländischer Schülerinnen und Schüler auch in der Sekundastufe II ihr Ende noch nicht gefunden hat, sondern lediglich in etwas abgedämpfter Weise weiter verläuft. Welche Sachverhalte treffen nun für die Schülerinnen und Schüler der Sekundarstufe II zu?

\section{Fragestellung, Hypothesen und Datenbasis}

Fragestellung: Wir verfolgen die Bildungsbeteiligung der ausländischen Jugendlichen auf folgenden vier Ebenen: 
- Umfang der Bildungsbeteiligung in den unterschiedlichen Typen der Sekundarstufe II;

- Entwicklung der Bildungsbeteiligung über die Periode von 20 Jahren;

- Erschwerung der Zugänge zum Bildungssystem der Sekundarstufe II;

- Das letzte Kapitel behandelt die Berufslehren in besonderer Weise: Es wird untersucht, ob sich die Anspruchsniveaus der gewählten Berufe (BAN) zwischen den Jugendlichen unterschiedlicher ethnolinguistischer Herkunft unterscheiden. Spezifisch wird die Kompensationshypothese überprüft, welche besagt, dass die ausländischen Jugendlichen, welche aufgrund der schulsprachlich dominierten Auslese am Ende der Primarstufe einem Schultyp der Sekundarstufe I mit (zu) niedrigen Ansprüchen zugewiesen wurden, diese Benachteiligung zumindest bei der Berufswahl 3 bis 4 Jahre später kompensieren können, indem sie nun Berufe mit einem relativ hohen beruflichen Anspruchsniveau $B A N$ (bezüglich intellektueller und schulisch-leistungsmässiger Erfordernisse) ergreifen.

Datenbasis: Das Datenmaterial basiert auf den aktuellsten verfügbaren Gesamtstatistiken (August 2000) : Bei den gesamtschweizerischen Daten handelt es sich auf der Sek II um N = 297'609 Jugendliche (CH: 249'765; AUSL: 47'844); bei den 16-20-Jährigen um $\mathrm{N}=408$ '794 (CH: 322'080; AUSL: 86'714). Eine differenzierte Datenanalyse, welche die Parameter Geschlecht und Ausbildungstypus auf der SEK II verbindet, ist erst seit dem Schuljahr 1998/99 für wenige Kantone möglich. Ich stütze mich dabei auf die differenzierten Daten des Kantons Bern, d.h. auf eine Population von 167'796 Schülerinnen und Schülern insgesamt und ein Sample von 42'554 AbsolventInnen der bernischen Sek II (CH ${\text { FrAU: 19'164; } \text { CH }_{\text {MAN: }} \text { 20'586; AUSL }}_{\text {FrAU: 1'302; AUSL }}$ MAN: 1'5024. Eine Gliederung in andere soziologische Parameter (wie sozio-ökonomische Zugehörigkeit oder Zuwanderungszeitpunkt) ist aufgrund dieser Datenbasis nicht möglich.

\section{Die Bildungsbeteiligung ausländischer und schweizerischer Jugendlicher}

\section{Bildungsbeteiligung ausländischer und schweizerischer Jugendlicher auf der Sekundarstufe II im Vergleich}

In Hinblick auf den erwähnten bildungsökonomischen Gesichtspunkt ist zunächst einmal von Interesse, ob und in welchem Masse Jugendliche nach der Absolvierung ihrer obligatorischen Schulpflicht in das weiterführende Ausbildungssystem der Sekundarstufe II eingeschleust werden. Ganz generell ist zu sagen, dass die Schweiz im internationalen Vergleich über einen erfreulich hohen Bildungsstand verfügt: $80 \%$ der Wohnbevölkerung verfügt über eine nachobligatorische Ausbildung, womit sie das OECD-Mittel (40\%), aber auch dasjenige 
der meisten EU-Staaten oder der Nachbarländer mit Ausnahme von Deutschland (82\%) weit übertrifft (BfS, 1999, S. 14f.). Unqualifizierte Arbeitskräfte sind zwar von einem gewissen Interesse für die Wirtschaft, sie gehören aber gleichzeitig auch zu derjenigen Gruppe, welche in wirtschaftlichen Krisen der Öffentlichkeit zuerst zur Last fällt. Zudem stehen die Ressourcen unqualifizierter Arbeitnehmerinnen und Arbeitnehemern in einer schnell sich wandelnden Wissenswelt nicht zur Verfügung, weil grundlegende Schlüsselqualifikationen nicht gefördert bzw. ausgebildet wurden. Ganz generell kann gesagt werden, dass darum das Bemühen der Bildungsverantwortlichen dahin geht, dass möglichst viele Jugendliche eine Sekundarstufe II (Sek II) absolvieren5.

(a) Niedrige Bildungsbeteiligung ausländischer Schülerinnen und Schüler, insbesondere der Nicht-Anrainerstaaten und Nicht-EU-Staaten.

In der Tab. 1 wird die Bildungsbeteiligung aufder Sekundarstufe II dargestellt. Die Prozentangaben ohne Klammern beziehen auf die Gesamtbevölkerung der 16bis 20-Jährigen, die Prozentangaben in den Klammern haben als Vergleichs-population alle jene 16- bis 20-Jährigen, die mindestens 1 Jahr eine Schweizer Schule besuchten. Man kann die Sachverhalte wie folgt zusammenfassen:

1 Die Bildungsbeteiligungen der Schweizerinnen und Schweizer und Ausländerinnen und Ausländern unterscheiden sich sowohl im Ausmass als auch im eingeschlagenen Bildungsweg der Sekundarstufe II stark voneinander. Während 77.55\% der Schweizerinnen und Schweizer eine Sekundarstufe II-Ausbildung wählen, sind es bei den Ausländerinnen und Ausländern nur 55.17\%. Allerdings gilt auch hier die Regel, dass der längere Schulbesuch in der Schweiz, die Bildungschancen stark erhöht (Müller, 1997): Die Bildungsbeteiligung der ausländischen Jugendlichen steigt auf 71.64\% (Klammerwerte) an, wenn die ausländischen Schülerinnen und Schüler ein oder mehrere Jahre in der Schweiz beschult werden. Die Werte erscheinen niedrig. Es ist jedoch zu bedenken, dass 2-3 \% der Bevölkerung zu den beruflich nicht bildungsfähigen Menschen gehörten. Zudem wird ein Teil der Lehren zu einem späteren Zeitpunkt absolviert, so dass im Alter von 24 Jahren 92\% der Schweizerinnen und Schweizer, jedoch erst 77\% der Ausländerinnen und Ausländer eine SEK-II-Ausbildung angetreten haben (Lischer, 1997, S. 23).

2 Problematisch erweist sich die Bildungsbeteiligung für Jugendliche aus Nicht-EU-Staaten. So fallen die Angehörigen der ehemaligen jugoslawischen Republiken $(32.57 \%)$ und der Türkei $(32.13 \%)$ auch bei einem vorherigen Schulbesuch in der Schweiz stark ab. Die Bildungsbeteiligung beträgt hier lediglich ca. 39\%. Generell kann man davon ausgehen, dass bei Angehörigen von Anrainerstaaten (ohne Italien) eine hohe Bildungsbeteiligung besteht, gefolgt von den übrigen EU-Staaten, während die Jugendlichen aus Ex-Jugoslawien; Türkei und Albanien stark abfallen. 
Tab. 1: Bildungsbeteiligung auf der Sekundarstufe II bei Schweizerinnen und Schweizern, Ausländerinnen und Ausländern und ausgewäblten Nationen; Schuljahr 1998/99 (100\% = 16 bis 20-Jährige der jeweiligen Nation)

\begin{tabular}{|c|c|c|c|c|c|c|c|c|c|}
\hline & \multicolumn{7}{|c|}{ Ausbildungstyp auf der Sekundarstufe II } & \multicolumn{2}{|c|}{$\begin{array}{l}\text { Bildungsbeteili- } \\
\text { gungsquotient } \\
\text { BBQ }\left({ }^{* *}\right)\end{array}$} \\
\hline & SEK II & $\begin{array}{l}\text { Keine } \\
\text { SEK II }\end{array}$ & $\begin{array}{c}\text { Mittel- } \\
\text { schule } \\
(* * *)\end{array}$ & $\begin{array}{l}\text { Berufs- } \\
\text { lehre }\end{array}$ & DMS & Anlehe & $\begin{array}{c}\text { Andere } \\
\text { Ausbil- } \\
\text { dung }\end{array}$ & \multirow{2}{*}{$\begin{array}{l}\text { alle 16- } \\
\text { 20- } \\
\text { Jährigen }\end{array}$} & \multirow{2}{*}{$\begin{array}{l}\text { 16-20- } \\
\text { Jährigen } \\
\text { mit min. } \\
1 \text { Jahr } \\
\text { Schule } \\
\text { in CH }\end{array}$} \\
\hline Herkunft & $(*)$ & $(*)$ & & & & & & & \\
\hline Schweiz & $\begin{array}{l}77.55 \% \\
(77.55 \%)\end{array}$ & $\begin{array}{l}22.45 \% \\
(22.45 \%)\end{array}$ & $20.72 \%$ & $81.85 \%$ & $2.90 \%$ & $0.08 \%$ & $2.07 \%$ & $3 / 45$ & 3.45 \\
\hline Ausland & $\begin{array}{l}55.17 \% \\
(7 \times 4 \%)\end{array}$ & $\begin{array}{l}44.83 \% \\
(28.36 \%)\end{array}$ & $10.04 \%$ & $38.27 \%$ & $2.53 \%$ & $0.20 \%$ & $\%$ & 1.23 & 2.53 \\
\hline $\begin{array}{c}\text { Mittelmeerländer } \\
\text { (ohne Ex-YU) }\end{array}$ & $\begin{array}{l}29.70 / 2 \\
(66 / 98 \%)\end{array}$ & $\begin{array}{l}4 Q .30 \% \\
(33.02 \%)\end{array}$ & $8.44 \%$ & $44.29 \%$ & $3.18 \%$ & $0.13 \%$ & $3.79 \%$ & 1.48 & 2.03 \\
\hline $\begin{array}{l}\text { Lateinische } \\
\text { Länder }\end{array}$ & (7.63\% & $\begin{array}{l}32.37 \% \% \\
(27.05 \%)\end{array}$ & $10.68 \%$ & $49.23 \%$ & $3.81 \%$ & $0.12 \%$ & $3.91 \%$ & 2.09 & 2.70 \\
\hline $\begin{array}{l}\text { Nachbarländer } \\
\text { (ohne I) }\end{array}$ & $\begin{array}{c}96.63 \% \\
(-)\end{array}$ & $\begin{array}{l}3.37 \% \\
(-)\end{array}$ & $30.60 \%$ & $59.13 \%$ & $4.16 \%$ & $0.05 \%$ & $2.75 \%$ & 28.72 & - \\
\hline Italien & $\begin{array}{l}73.56 \% \\
(77.03 \%) \\
\end{array}$ & $\begin{array}{r}26.44 \% \\
(22.97 \%) \\
\end{array}$ & $10.96 \%$ & $57.16 \%$ & $2.88 \%$ & $0.08 \%$ & $2.55 \%$ & 2.78 & 3.35 \\
\hline $\begin{array}{c}\text { Ex-Jugos- } \\
\text { lawien }\end{array}$ & $\begin{array}{l}32.57 \% \\
(38.54 \%) \\
\end{array}$ & $\begin{array}{r}67.43 \% \\
(61.46 \%)\end{array}$ & $8.28 \%$ & $25.08 \%$ & $0.73 \%$ & $0.25 \%$ & $4.48 \%$ & 0.4 & 0.63 \\
\hline Türkei & $\begin{array}{l}32.13 \% \\
39.02 \%) \\
\end{array}$ & $\begin{array}{l}67.87 \% \\
(60.98 \%)\end{array}$ & $3.70 \%$ & $23.17 \%$ & $1.43 \%$ & $0.14 \%$ & 3.8 & 0.47 & 0.64 \\
\hline Alle & $\begin{array}{l}72 . \$ 0 \% \\
(71.64 \%\end{array}$ & $\begin{array}{r}27.20 \% \\
(28.36 \%) \\
\end{array}$ & $18.46 \%$ & $48.27 \%$ & $2.82 \%$ & $8 \%$ & $2.55 \%$ & & 0.39 \\
\hline
\end{tabular}

$\left(^{*}\right)$ Die Zahl ohne Klammer hat als Bezugsgrösse: alle 16-20-Jährigen $=100 \%$, d.h. inkl. jener, die nie die Schweizer Schule besucht haben. Die Zahl in der Klammer heisst: alle 16-20-Jährigen, welche 1 Jahr oder mehr die Schweizer Schule besuchten $=100 \%$.

${ }^{(* *)}$ Der BBQ errechnet sich aus dem Verhältnis von SEK II JA! zu SEK II NEIN!. Lesebeispiel: BBQ: Auf 1.23 ausländische Jugendliche in der SEK II fällt 1 ausländischer Jugendlicher ohne SEK IIAusbildung. Auf 1 SchweizerIn ohne Ausbildung fallen 3.45 mit Ausbildung!

${ }^{(* *)}$ Maturitätsschulen, Lehrerinnen- und Lehrerbildung und Berufsmaturitätsschulen.

Lesebeispiel: Vom Gesamtbestand aller in der Schweiz niedergelassenen ausländischen Jugendlichen im Alter von 16 bis 20 Jahren besuchten bei den SchweizerInnen 20.72\% eine Mittelschule, bei den Ausländerinnen und Ausländern nur 10.04\%. Bei den Ex-JugoslawInnen sind es gar nur 2.28\%.

3 Eingeschlagene Bildungswege / Schultypen: Bezüglich der Ausbildungsgänge lassen sich zwei generelle Feststellungen machen: (1) Die Ausländerinnen und Ausländer sind in den Mittelschulen und in den Berufslehren sehr stark untervertreten (Mittelschule: $\mathrm{CH}$ : 20.72\%, AUSL: 10.4\%; Berufslehren: $\mathrm{CH}$ : 
51.58\%, AUSL: $38.27 \%)$. (2) Die tertiär orientierten Bildungsgänge (Mittelschulen) werden im Wesentlichen durch Schweizerinnen und Schweizer besetzt. Bei den Unterrichtsberufen ist der Anteil ausländischer Jugendlicher äusserst niedrig: Im Schuljahr 1998/99 fallen z.B. im Kanton Bern auf 1647 Schweizer am Lehramt Studierende (4.1\% der Sekundarstufe II) gerade noch 12 ausländische $(0.4 \%$ der Sekundarstufe II) Studierende und dies bei einem Anteil von $14.8 \%$ ausländischen Volksschülerinnen und Schüler. Es lässt sich somit von einer massiven Unterrepräsentanz der Ausländerinnen und Ausländern in den Mittelschulen sprechen. Für die Jugendlichen aus der Türkei (2.28\%) und aus Ex-Jugoslawien (3.7\%) ist die Situation prekär. Etwas weniger ausgeprägt fällt der Vergleich bei den Berufslehren aus. Hier sind die Mittelmeerländer immerhin zu 44.29\% vertreten. Die Jugendlichen aus der Türkei und Ex-Jugoslawien sind mit Werten von $23 \%$ bis $25 \%$ immer noch stark untervertreten.

4 Der in den zwei letzten Spalten dargestellte Bildungsbeteiligungsquotient $B B Q$ gibt den jetzigen Zustand zusammenfassend gut wieder: Während bei den Schweizerinnen und Schweizern auf 1 nicht ausgebildete/n Jugendliche/n 3.45 Ausgebildete fallen, so sind es bei den Ausländerinnen und Ausländern nur 1.23, bei den TürkInnen und Ex-JugoslawInnen gar nur 0.48 Jugendliche, d.h. siebenmal weniger! Dabei handelt es sich hier um die Angehörigen mit bleibendem Aufenthaltsrecht und nicht etwa um AsylbewerberInnen, die in dieser Statistik gar nicht auftreten.

\section{(b) Repräsentanz der SEK-II-AbsolventInnen verschiedener Herkunft an Mittel- schulen}

In Tab. 2 werden die proportionalen Anteile der SEK II-AbsolventInnen mit Bezug zum einzelnen Ausbildungsgang $(=100 \%)$ dargestellt. Dies ermöglicht, den theoretischen Erwartungswert (3. Spalte) für je die Gesamtgruppe der AUSL und der $\mathrm{CH}$ mit den empirischen Anteilen in den 5 Ausbildungsgängen (4. bis 8. Spalte) zu vergleichen und das Prozentmass der Unter-bzw. Überrepräsentanz festzulegen (letzte Spalte). Gesamtschweizerisch beträgt in den Mittelschulen der Anteil der AUSL lediglich $11.5 \%$ bei einer Erwartung von 16.1\%. Die AUSL sind somit - gemessen an ihrer Gesamtbevölkerung - zu 28.2\% in den Mittelschulen untervertreten, während die Schweizer Jugendlichen zu 5.4\% übervertreten sind.

Gravierend sieht die Situation für alle Angehörigen des nicht französisch oder deutsch sprechenden Auslandes aus. Selbst für die als sprachlich und kulturell gut integriert geltenden ItalienerInnen beträgt die Unterrepräsentanz an den Mittelschulen 41.3\%! Am extremsten sind die Diskrepanzen wiederum für die Angehörigen des ehemaligen Jugoslawien und der Türkei; hier sind die tatsächlichen Anteile in den Mittelschulen 2- bis 4-mal tiefer als der Erwartungswert. In der Tatsache der Überrepräsentanz von Schweizerinnen und Schweizern und Angehörigen der deutsch und französisch sprechenden Nachbarländer in den 
Mittelschulen im Unterschied zu den übrigen Ländern lässt sich leicht die Wirkung des monolingualen Habitus der Schule erkennen.

Bei den Berufslehren entsprechen die relativen Anteile einigermassen den erwarteten Werten. Die Statistik zeigt auch, dass die ausländischen Jugendlichen vermehrt auf «alternative» Lösungen ausweichen, sofern Sie nicht in einer Mittelschule oder Berufslehre Zugang gefunden haben: Es sind dies im geringeren Masse die Diplommittelschulen, besonders aber die Anlehren und die Vorlehren und die Zwischenjahr-Lösungen (z.B. 10. Schuljahr) ${ }^{6}$. Hier zeigt sich, dass die ausländischen Schülerinnen und Schüler viel daran setzen, in einem zweiten Anlauf den Zugang zu den Berufslehren zu schaffen.

Tab. 2: Prozentuale Anteile bei Schweizerinnen und Schweizern, Ausländerinnen und Ausländern und ausgewählten Nationen in den verschiedenen Bildungstypen der Sekundarstufe II; Schuljahr 1998/99

\begin{tabular}{|c|c|c|c|c|c|c|c|c|}
\hline & \multicolumn{2}{|c|}{$\begin{array}{l}\text { Bezug alle 16- } \\
\text { 20-Jährigen } \\
=100 \%\end{array}$} & \multicolumn{6}{|c|}{$\begin{array}{l}\text { Bezug alle SEK II des jeweiligen Ausbildungsganges } \\
\qquad=100 \%\end{array}$} \\
\hline Ausbildung & $\begin{array}{l}\text { Keine } \\
\text { SEK II }\end{array}$ & $\begin{array}{l}\text { SEK II } \\
\text { (Erwar- } \\
\text { tungs- } \\
\text { wert) }\end{array}$ & $\begin{array}{l}\text { Mittel- } \\
\text { schule }\end{array}$ & $\begin{array}{l}\text { Berufs- } \\
\text { lehre }\end{array}$ & DMS & Anlehre & Andere & $\begin{array}{c}\text { Unter-/ } \\
\text { Über- } \\
\text { reprä- } \\
\text { sentanz } \\
\text { in Mittel- } \\
\text { schulen }\end{array}$ \\
\hline \multicolumn{9}{|l|}{ Herkunft } \\
\hline Schweiz & $65 \%$ & $83.9 \%$ & $88.5 \%$ & $83.4 \%$ & $81.0 \%$ & $58.2 \%$ & $64.3 \%$ & $5.4 \%$ \\
\hline Ausland & $35.0 \%$ & $16.1 \%$ & $11.5 \%$ & $16.6 \%$ & $19.0 \%$ & $41.8 \%$ & $35.7 \%$ & $-28.2 \%$ \\
\hline $\begin{array}{l}\text { Mittelmeerländer } \\
\text { (ohne Ex-YU) }\end{array}$ & $14.5 \%$ & $8.0 \%$ & $4.5 \%$ & $8.8 \%$ & $11.0 \%$ & $12.0 \%$ & $14.6 \%$ & $-44.3 \%$ \\
\hline $\begin{array}{l}\text { Lateinische } \\
\text { Länder }\end{array}$ & $9.9 \%$ & $7.7 \%$ & $4.8 \%$ & $8.4 \%$ & $11.2 \%$ & $10.1 \%$ & $12.8 \%$ & $-37.7 \%$ \\
\hline $\begin{array}{l}\text { Nachbarländer } \\
\text { (ohne I) }\end{array}$ & $0.2 \%$ & $1.8 \%$ & $2.2 \%$ & $1.6 \%$ & $2.0 \%$ & $0.7 \%$ & $1.5 \%$ & $24.9 \%$ \\
\hline Italien & $4.0 \%$ & $4.2 \%$ & $2.5 \%$ & $4.8 \%$ & $4.2 \%$ & $3.1 \%$ & $4.2 \%$ & $-41.3 \%$ \\
\hline $\begin{array}{l}\text { Ex-Jugos- } \\
\text { lawien }\end{array}$ & $16.8 \%$ & $3.0 \%$ & $0.8 \%$ & $3.5 \%$ & $1.8 \%$ & $16.8 \%$ & $11.7 \%$ & $-72.4 \%$ \\
\hline Türkei & $4.6 \%$ & $0.8 \%$ & $0.4 \%$ & $0.9 \%$ & $0.9 \%$ & $2.6 \%$ & $2.8 \%$ & $-54.5 \%$ \\
\hline Alle & $100 \%$ & $100 \%$ & $100 \%$ & $100 \%$ & $100 \%$ & $100 \%$ & $100 \%$ & $0 \%$ \\
\hline
\end{tabular}

Lesebeispiel: Italien: Von einem erwarteten Wert von 4.2\% der Italienerinnen und Italiener befinden sich $2.5 \%$ in der Mittelschule. Bei den Schweizerinnen und Schweizern sind hingegen bei einem erwarteten Wert von $83.9 \% 88.5 \%$ in der Mittelschule. Die Italienerinnen und Italiener sind mit $41.3 \%$ an der Mittelschule untervertreten.

(c) Keine generelle Unterrepräsentanz in höheren Ausbildungsgängen bei ausländischen Frauen innerhalb der Sekundastufe II (Tab. 3):

Im Unterschied zur manchmal geäusserten Meinung, kann weder von einer Benachteiligung der ausländischen noch der schweizerischen Frauen innerhalb der 
Ausbildungstypen der Sekundarstufe II gesprochen werden. Einschränkend sei allerdings gesagt, dass die Statistik sich nur auf jene Jugendliche bezieht, welche die Sekundarstufe II absolvieren. Jene 44.83\% Ausländerinnen und Ausländer und $22.45 \%$ Schweizerinnen und Schweizer (Tab. 1), die keine Sekundarstufe II absolvieren, bleiben aus der Analyse ausgeschlossen.

Mittelschulen und Berufslehren: So betrachtet sind die Frauen aller hier analysierten Nationen in den tertiär orientierten Mittelschulen im Vergleich zu den Männern im Verhältnis von ca. 3:2 stark übervertreten, in den Berufsschulen hingegen untervertreten (Tab. 3). Geht man von der generalisierenden Annahme aus, dass ein Mittelschulbesuch der Berufslehre bezüglich des schulischen Anspruchsniveaus übergeordnet ist, so kann gar von einem höheren Ausbildungsniveau bei ausländischen und inländischen Frauen im Vergleich zu ihren männlichen Landsleuten gesprochen werden.

Berufsmaturitätsschulen - obwohl 1998/99 noch mit sehr geringen Anteilen vertreten - scheinen eher die Angelegenheit von Männern zu sein. Die relativen Anteile von AUSL und CH sind stark ausgeglichen (CH: 0.9\%; AUSL: 0.7\%). Es ist möglich, dass bereits hier eine Art Kompensationseffekt für «verpasste Bildungschancen" zugunsten der AUSL wirksam ist (vgl. letztes Kapitel, S. 69). Dass Frauen überdurchschnittlich in Berufslehren (unter 3 Jahren) vertreten sind, welche als Voraussetzung zur Berufsmaturität nicht anerkannt sind (Borkowsky, 2000; Grossenbacher, 2000), mag ihren geringen Anteil in den Berufsmaturitätsschulen mit erklären.

Übrige SEK II: In Hinblick auf die Geschlechterverteilung lässt sich festhalten: Bei allen Nationen (mit Ausnahme der Nachbarländer ohne Italien) ist die Diplommittelschule DMS hauptsächlich eine Schule der Frauen, was damit zusammenhängen mag, dass diese primär auf sog. "Frauenberufe» vorbereiten (KrankenpflegerInnen, LaborantInnen, SozialarbeiterInnen usf.). Umgekehrt sind Anlehren und Vorlehren in der Regel klar durch Männer dominiert, und dies in ausgeprägterer Form bei den AUSL. Uneinheitlich präsentiert sich das Bild bei der Lösung "Zwischenjahr». Die Frauen scheinen diese Möglichkeit leicht vermehrt zu nutzen als die Männer.

Zusammenfassend ergibt sich auch bei der Bezugsgruppe der Jugendlichen (16- bis 20-Jährige und/oder Sek II-AbsolventInnen) jenes Bild, das man bereits von der obligatorischen Volksschule her kennt: Bildungsmässige Benachteiligungen entlang der Linie der ethnolinguistischen Zugehörigkeit (Ausländer vs. Schweizer) sind weit ausgeprägter als jene entlang der Linie der Gender (vgl. für die Volksschule: Müller, 1997). Gewissermassen bildet sich eine Art Hierarchie der Benachteiligung in der Kombination der soziologischen Parameter ethnolinguistische Zugehörigkeit und Gender: 1. Schweizer Frauen; 2. Schweizer Männer; 3. ausländische Frauen, 4. ausländische Männer und unter den grösseren Nationen die Abfolge: 1. Schweizerinnen und Schweizer und Angehörige von Anrainerstaaten (ohne Italien); 2. Angehörige lateinischer Länder des EU-Raums (ohne Frankreich); 3. TürkInnen, Ex-JugoslawInnen und AlbanerInnen. 
Tab. 3: Anteile der Jugendlichen auf der SEK II des Kantons Bern aus verschiedenen Nationen nach Geschlecht unterschieden (100\% = SEK II Absolventen der jeweiligen Nation und des jeweiligen Geschlechtes; NTotal: 44'554; $\mathrm{CH}_{\text {weiblich: }}$

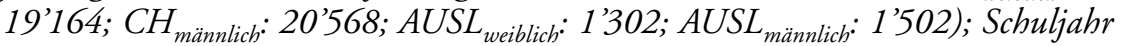
$1998 / 99$.

\begin{tabular}{|c|c|c|c|c|c|c|c|c|c|c|c|}
\hline & & \multicolumn{4}{|c|}{ Mittelschule } & \multirow{2}{*}{ 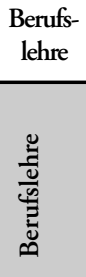 } & \multicolumn{5}{|c|}{ Übrige SEK II } \\
\hline Herkunft & 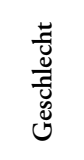 & 莺 & مُ & 皇: & 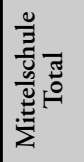 & & $\sum_{0}^{\infty}$ & 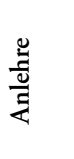 & 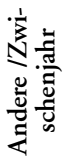 & 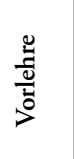 & 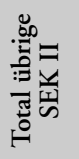 \\
\hline \multirow[t]{3}{*}{ Schweiz } & MAN & $14.4 \%$ & $1.8 \%$ & $1.2 \%$ & $17.4 \%$ & $79.0 \%$ & $0.8 \%$ & $1.3 \%$ & $1.0 \%$ & $0.5 \%$ & $3.6 \%$ \\
\hline & FRAU & $18.4 \%$ & $6.7 \%$ & $0.5 \%$ & $25.6 \%$ & $68.7 \%$ & $2.9 \%$ & $0.7 \%$ & $1.9 \%$ & $0.3 \%$ & $5.8 \%$ \\
\hline & Insges. & $16.4 \%$ & $4.1 \%$ & $0.9 \%$ & $21.4 \%$ & $74.0 \%$ & $1.8 \%$ & $1.0 \%$ & $1.4 \%$ & $0.4 \%$ & $4.6 \%$ \\
\hline \multirow[t]{3}{*}{ Ausland } & MAN & $10.7 \%$ & $0.3 \%$ & $0.9 \%$ & $11.9 \%$ & $80.8 \%$ & $1.1 \%$ & $2.6 \%$ & $2.3 \%$ & $1.3 \%$ & $7.3 \%$ \\
\hline & FRAU & $15.2 \%$ & $0.6 \%$ & $0.4 \%$ & $16.2 \%$ & $76.2 \%$ & $3.1 \%$ & $0.9 \%$ & $2.8 \%$ & $0.7 \%$ & $7.5 \%$ \\
\hline & Insges. & $12.8 \%$ & $0.4 \%$ & $0.7 \%$ & $13.9 \%$ & $78.7 \%$ & $2.0 \%$ & $1.8 \%$ & $2.6 \%$ & $1.0 \%$ & $7.4 \%$ \\
\hline \multirow{3}{*}{$\begin{array}{c}\text { Mittel- } \\
\text { meer- } \\
\text { länder } \\
\text { I,E,P,GR }\end{array}$} & MAN & $7.9 \%$ & $0.3 \%$ & $1.3 \%$ & $9.5 \%$ & $85.4 \%$ & $0.2 \%$ & $2.6 \%$ & $1.6 \%$ & $0.7 \%$ & $5.1 \%$ \\
\hline & FRAU & $12.0 \%$ & $0.6 \%$ & $0.6 \%$ & $13.2 \%$ & $81.1 \%$ & $3.6 \%$ & $0.0 \%$ & $1.8 \%$ & $0.2 \%$ & $5.6 \%$ \\
\hline & Insges. & $9.8 \%$ & $0.5 \%$ & $1.0 \%$ & $11.3 \%$ & $83.5 \%$ & $1.7 \%$ & $1.4 \%$ & $1.7 \%$ & $0.5 \%$ & $5.3 \%$ \\
\hline \multirow{3}{*}{$\begin{array}{c}\text { Nach- } \\
\text { bar- } \\
\text { länder } \\
\text { (ohne I) }\end{array}$} & MAN & $17.3 \%$ & $0.7 \%$ & $0.7 \%$ & $18.7 \%$ & $69.1 \%$ & $7.9 \%$ & $2.9 \%$ & $0.7 \%$ & $0.7 \%$ & $12.2 \%$ \\
\hline & FRAU & $22.2 \%$ & $0.8 \%$ & $0.0 \%$ & $23 \%$ & $68.3 \%$ & $7.1 \%$ & $0.8 \%$ & $0.8 \%$ & $0.0 \%$ & $8.7 \%$ \\
\hline & Insges. & $19.6 \%$ & $0.8 \%$ & $0.4 \%$ & $20.8 \%$ & $68.7 \%$ & $7.5 \%$ & $1.9 \%$ & $0.8 \%$ & $0.4 \%$ & $10.6 \%$ \\
\hline \multirow[t]{3}{*}{ Italien } & MAN & $8.8 \%$ & $0.5 \%$ & $1.9 \%$ & $11.2 \%$ & $86.2 \%$ & $0.0 \%$ & $1.9 \%$ & $0.5 \%$ & $0.3 \%$ & $2.7 \%$ \\
\hline & FRAU & $12.5 \%$ & $0.7 \%$ & $0.3 \%$ & $13.5 \%$ & $82.2 \%$ & $2.7 \%$ & $0.0 \%$ & $1.7 \%$ & $0.0 \%$ & $4.4 \%$ \\
\hline & Insges. & $10.4 \%$ & $0.6 \%$ & $1.2 \%$ & $12.2 \%$ & $84.4 \%$ & $1.2 \%$ & $1.0 \%$ & $1.0 \%$ & $0.1 \%$ & $3.3 \%$ \\
\hline \multirow[t]{3}{*}{ Türkei } & MAN & $4.9 \%$ & $0.0 \%$ & $1.2 \%$ & $6.1 \%$ & $87.7 \%$ & $0.0 \%$ & $0.0 \%$ & $3.7 \%$ & $2.5 \%$ & $6.2 \%$ \\
\hline & FRAU & $12.6 \%$ & $2.1 \%$ & $0.0 \%$ & $14.7 \%$ & $82.1 \%$ & $1.1 \%$ & $0.0 \%$ & $2.1 \%$ & $0.0 \%$ & $3.2 \%$ \\
\hline & Insges. & $9.1 \%$ & $1.1 \%$ & $0.6 \%$ & $10.8 \%$ & $84.7 \%$ & $0.6 \%$ & $0.0 \%$ & $2.8 \%$ & $1.1 \%$ & $4.5 \%$ \\
\hline \multirow{3}{*}{$\begin{array}{l}\text { Ex-Jugos- } \\
\text { lawien u. } \\
\text { Albanien }\end{array}$} & MAN & $7.3 \%$ & $0.0 \%$ & $0.3 \%$ & $7.6 \%$ & $80.5 \%$ & $0.3 \%$ & $3.6 \%$ & $5.6 \%$ & $2.3 \%$ & $11.8 \%$ \\
\hline & FRAU & $9.3 \%$ & $0.0 \%$ & $0.3 \%$ & $9.6 \%$ & $82.0 \%$ & $1.7 \%$ & $3.0 \%$ & $2.3 \%$ & $1.3 \%$ & $8.3 \%$ \\
\hline & Insges. & $8.3 \%$ & $0.0 \%$ & $0.3 \%$ & $8.6 \%$ & $81.2 \%$ & $1.0 \%$ & $3.3 \%$ & $4.0 \%$ & $1.8 \%$ & $10.1 \%$ \\
\hline
\end{tabular}

Chi-Quadrat über Insgesamt x Schweiz/Ausland: 185.2; p = 0.000; df: 7.

Lesebeispiel: In den Mittelschulen ist die Gruppe der SchweizerInnen insgesamt gegenüber den AusländerInnen übervertreten. Das Verhältnis zwischen den Geschlechtern hingegen ist bei beiden Gruppen etwa gleich: Schweizerinnen zu Schweizern $=25.6 \%$ zu 17.4\%, bei den AusländerInnen 16.2\% zu $11.9 \%$. Auf 2 Mittelschüler fallen also ca. 3 Mittelschülerinnen. 


\section{Die Entwicklung der Bildungsbeteiligung über die \\ Periode von 20 Jahren (1980/81 bis 1998/99)}

Unter bildungsökonomischen Gesichtspunkten ist der Zusammenhang von Bildungsinvestitionen und Bildungserträgen von zentraler Bedeutung. 1995 wurden in der Schweiz 21 Milliarden Sfr (5.6\% des BIP oder ca. 15\% der öffentlichen Ausgaben) in die Bildung investiert. Damit steht die Schweiz bezüglich der öffentlichen Bildungsausgaben mit den USA an der Spitze aller OECD-Länder, also auch vor den Nachbarländern (D: 9.6\%; I: 9\%; F: 11\%; AU: 10.7\%). Der indexierte Anstieg der Bildungsausgaben von 1980 bis 1996 beträgt 42\% (BfS, 1999). Allerdings ist Vorsicht geboten, solche Zahlen in direkte Abhängigkeit zur zunehmenden Bildungsbeteiligung in den einzelnen Schultypen zu bringen, da auch demografische (z.B. Zuwanderung), wirtschaftliche (z.B. Rezession) oder bildungspolitische (z.B. Bildungsinvestitionsschwerpunkte) Entwicklungen hier mitspielen (vgl. zur Diskussion: Alsalam et al., 1995; Psacharopoulos, 1987, 1994; OECD, 1998). Es lassen sich aber zwei Aspekte festhalten, die für die jetzige Diskussion von Bedeutung sind: (1) In der zwanzigjährigen Periode von $1980 / 81$ bis 1998/99 ist auch die Bildungsbeteiligung, bezogen auf die Gesamtpopulation der 16-20-Jährigen, um über 13\% gestiegen (von 59.56\% auf $72.8 \%$ ). Dabei haben die Mittelschulen mit $6.5 \%$ viel stärker zugelegt als die Berufsschulen (4.3\%) ( $A b b .1$ ). (2) Der Bildungsbedarf an höheren SEK-II-Abschlüssen hat in der gleichen Periode von 1.56 Schülerinnen und Schüler auf 2.02 zugenommen (Müller, 2000, S. 75f.; 2001, S. 44). Zweifellos spiegelt sich in beiden Aspekten nicht nur der zunehmende gesellschaftliche Bedarf an besser bzw. länger ausgebildeten Menschen, sondern auch der technologische Wandel der Arbeitswelt wider. Werden also von diesem Anstieg alle Jugendlichen unabhängig ihres ethnolinguistischen Hintergrunds gleichermassen profitieren?

(1) Entwicklung der Bildungsbeteiligung (Abb. 1): Bei der Gruppe der 16-20Jährigen ziehen die Schweizerinnen und Schweizer den höchsten Nutzen: Sie steigern ihre Beteiligung um 16\%, wovon über die Hälfte, 8.2\%, auf die Mittelschulen und «nur» 5.5\% auf die Berufslehren entfallen. Bei den Ausländerinnen und Ausländern beträgt die Gesamtsteigerung 7.7\%, obwohl ihr «Nachholbedarf» bedeutend grösser war bzw. ist. Die Anteile an ausländischen Mittelschülerinnen und -schülern steigen in diesen 20 Jahren lediglich um 0.54\%. Ihr Zuwachs ist auf Berufslehren (4.8\%) und Anlehren zurückzuführen. Einzelne Nationen: Generell steigert sich der Anteil bei allen Nationen, dies allerdings in einer heterogenen Weise. Soweit es sich nicht um Schweizerinnen und Schweizer handelt, vermögen nur die Jugendlichen aus deutsch und französisch sprechenden Nachbarländern ihre Anteile an den Mittelschulen zu steigern (insgesamt: 29.48\%; Mittelschulen: 12.64\%; Berufslehren: 16.27\%; nicht dargestellt). Bei allen andern Ländern ist die Zunahme im Wesentlichen auf die Zunahme bei den Berufslehren zurückzuführen. Bei den Mittelmeerländern beträgt diese 17.95\% (Mittelschulen: 2.69\%; Berufslehren: 10.27\%), bei den als 
gut integriert geltenden ItalienerInnen gar 26\%, wobei auch hier die Steigerung in den Mittelschulen unterdurchschnittlich ausfällt (Mittelschulen: 4.58\% ; Berufslehren: $17.77 \%$ ). Für die Jugendlichen aus den ehemaligen Staaten von Jugoslawien fällt die Zunahme mit 7.88\% vergleichsweise bescheiden aus. Ihr Anteil ist an den Mittelschulen sogar um $-4.58 \%$ gesunken, und dies bei einer ohnehin sehr niedrigen Bildungsbeteiligung von 32.5\%. Betrachtet man die Ergebnisse im Überblick, so kann man zwar insgesamt von einer vermehrten Bildungsbeteiligung $(+13.24 \%)$ sprechen, jedoch geschah dies in den vergangenen 20 Jahren im mehr als doppeltem prozentualem Umfang zugunsten der Schweizerinnen und Schweizer. Pauschalisierend ausgedrückt heisst das: Das schweizerische Bildungssystem deckt seit 20 Jahren den Ausbildungsbedarf vor allem mit Rückgriff auf Schweizerinnen und Schweizer und greift in einem vergleichsweise viel geringeren Grad auf das Reservoir von Ausländerinnen und Ausländern zurück. Dieser Sachverhalt verschärft sich, wenn man bedenkt, dass fast der ganze anwachsende Bildungsbedarf an den Mittelschulen unter dem Ausschluss der statistischen Population der Ausländerinnen und Ausländer (+0.54\%!) geschehen ist. Bezüglich des Anspruchsniveaus der eingeschlagenen Ausbildungsgänge der SEK II öffnet sich hier eine Ausbildungsschere zwischen Schweizerinnen und Schweizern und Angehörigen von Anrainerstaaten, die in ihrer Herkunftssprache beschult werden (D; AU; FL; F) einerseits, und den Jugendlichen, welche selber (oder deren Eltern) über eine andere Herkunftssprache als die Schulsprache verfügen, andererseits.

(2) Sprachlich-nationale Präferenzen beim Decken des Bildungsbedarfs: Wenn wie behauptet - der Selektionsmechanismus primär via (schul-)sprachlicher Selektion verläuft, werden jene Ausländerinnen und Ausländer, bei welchen die Herkunftssprache mit dem lokalen Standard übereinstimmt (D, A, FL und F), ihre Bildungsquote ähnlich wie die Schweizerinnen und Schweizer steigern können, während dies bei den übrigen ethnolinguistischen (fremdsprachigen) Gruppen nicht oder in geringerem Masse der Fall sein wird. In $A b b$. 2 wurden gemäss diesem Kriterium die Indizes für den Mittelschulbesuch und die Berufslehren über die gleiche Verlaufsperiode für die drei Gruppen (1) CH, (2) D/F/A/FL und (3) alle Mittelmeerstaaten (ohne F) separat errechnet. Die Behauptung wird vollständig bestätigt. Im Verlaufe der 20-jährigen Periode steigt der Bildungsbeteiligungsindex bei den $\mathrm{CH}$ und den Anrainerstaaten (D, A, FL, F, ohne I) sehr stark an. Am Ende der Periode besuchen relativ betrachtet 1.3-mal mehr Schweizerinnen und Schweizer eine Mittelschule, bei den Anrainerstaaten (ohne I) sind es ca. 1.2-mal mehr. Zurückgegangen sind hingegen die Werte bei den (anderssprachigen) Mittelmeerländern (I/E/P/GR/TK/Ex-YU). Sie sind auf 90 Punkte abgesunken. 
Bildungsbeteiligung

Gesamtpopulation (AUSL. u. CH)

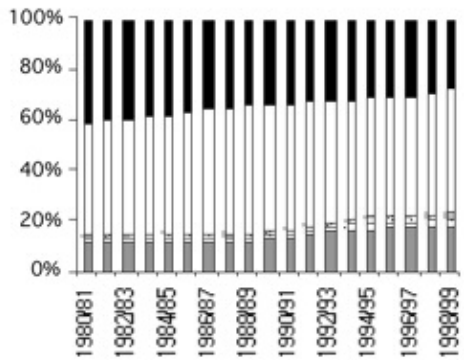

Bildungsbeteiligung

SchweizerInnen

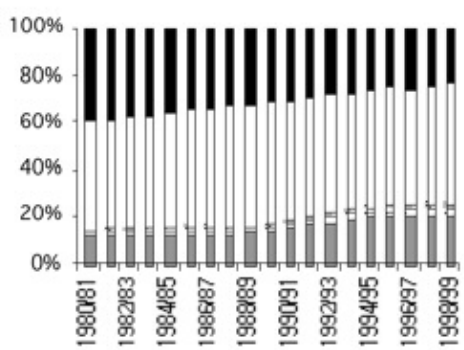

Bildungsbeteiligung

I/E/P/GR/TK (ohne Ex-JU)

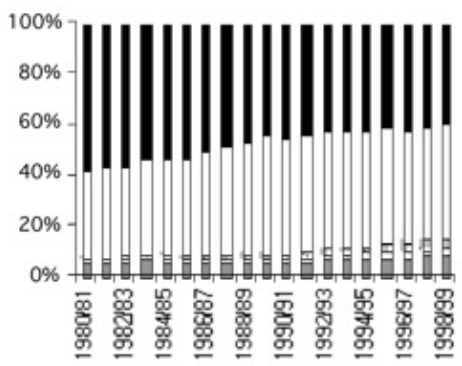

Bildungsbeteiligung

AusländerInnen

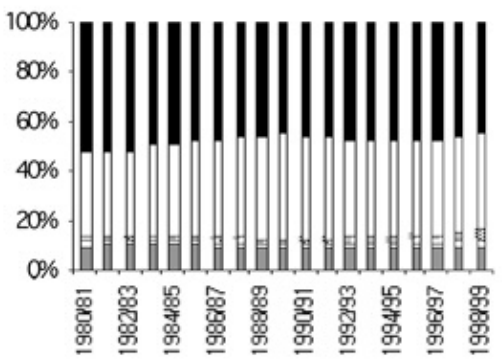

Bildungsbeteiligung

Ex-Jugoslawien

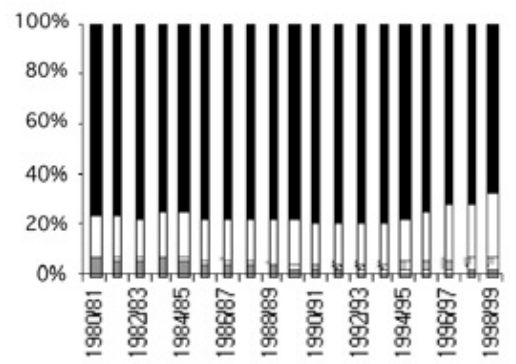

Bildungsbeteiligung ItalienerInnen

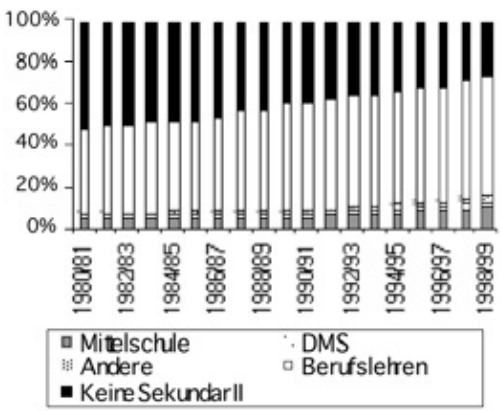

Abb. 1: Bildungsbeteiligung von 16- bis 20-jährigen Jugendlichen in den Jahren 1980/81 bis 1998/99 für verschiedene Nationen und Schultypen der SEK II (= Beruflehren / Mittelschulen / DMS / Andere); (Oberer schwarzer Balkenteil = SEK II Nein!; darunter weisse und graue Balkenteile = SEK II Ja!; Legende: s.u.). 


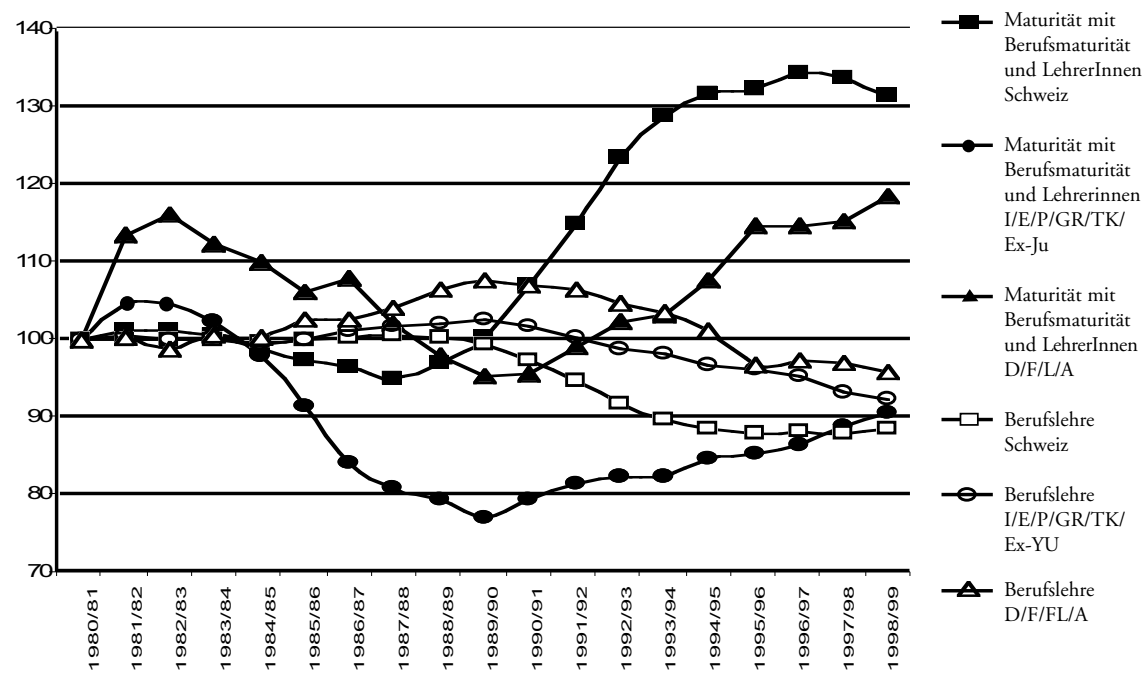

Abb. 2: Indizes für den Mittelschulbesuch und die Beruflehre von Schweizerinnen und Schweizern, Jugenlichen aus Mittelmeerländern (ohne F) und Nachbarländern F/D/A/FL (1980-1999) (100 Indexpunkte: 1980/81) (Bezug SEK II-Schüler)

Es trifft zu, dass hier die Angehörigen des ehemaligen Jugoslawiens stark ins Gewicht fallen. Ihr Index sinkt nämlich auf ca. 25 Punkte ab. Umgekehrt vermag das stark vertretene Italien mit einer Steigerung auf 111 Punkte dieses Manko nicht wettzumachen. Verkürzt man allerdings die Beobachtungsperiode um 10 Jahre, so sieht die Entwicklung etwas anders aus. Der Bedarf an Mittelschulabsolventinnen und -absolventen wird ab dem Schuljahr 1989/90 zum Teil auch mit Rückgriff auf die Angehörigen der Mittelmeerstaaten gedeckt. Bei genauerer Betrachtungsweise steigen seit diesem Zeitpunkt deren Indizes (+11 Punkte) an. Dies trifft jedoch in noch stärkerem Masse auch für die Schweizerinnen und Schweizer (+31 Punkte) und die Anrainerstaaten ohne Italien (+22 Punkte) zu. In den letzten drei Jahren scheint sich der Zuwachs bei den Schweizerinnen und Schweizern allerdings zu bremsen, während er bei den AUSL leicht, aber kontinuierlich anwächst. Berufslehren: Schliesslich bewegen sich die Indizes bei den monolingualen Schweizerinnen und Schweizern und Jugendlichen aus Anrainerstaaten gegenläufig zum Mittelschulbesuch. Bei den fremdsprachigen Ausländerinnen und Ausländern hingegen sind auch die Berufslehren um ca. 7 Punkte $(=-6.3 \%)$ abgefallen. Die genauere Datenanalyse ergibt, dass dieses Absinken durch den vermehrten Zufluss zu den An- und Vorlehren (+ 4.1\%) und Zwischenlösungen $(+3.7 \%)$ bedingt wird, Wege die sich eher dann anbieten, wenn die Lehrstellensuche erfolglos war. 


\section{Die Erschwerung der Zugänge zum Bildungssystem der SEK II}

Der Selektionsmechanismus der monolingualen Schule spiegelt natürlich nicht nur einen (schul-)sprachlichen Präferentialismus wider, sondern bildet, wie gesagt, einen Schutzmechanismus der majoritären monolingualen Gesellschaft gegenüber konkurrierenden Minoritätengruppen. Wie mächtig dieser Schutzmechanismus sein kann, zeigt sich, wenn man die Statistik von Schweizer und ausländischen Jugendlichen mit identischen schulischen Voraussetzungen im Übergang von der Sekundarstufe I zur Sekundarstufe II vergleicht. Man würde zumindest erwarten, dass gleiche schulische Voraussetzungen für alle Schülerinnen und Schüler unabhängig ihrer ethnolinguistischen Herkunft die gleichen Chancen bedeuteten.

Vorausgeschickt sei, dass im statistischen Durchschnitt die ausländischen Schülerinnen und Schüler, sind sie einmal einer Sekundarstufe I zugeteilt (z.B. $\mathrm{zu}$ einem der drei Leistungsniveaus Oberschule (=niedrig); Realklasse (=mittel), Sekundarschule $(=h o c h)^{7}$, in der Mathematik und den Fremdsprachen durchschnittlich höhere Leistungen als die monolingualen schweizerischen Kameraden erbringen, aber weiterhin niedrigere in der Schulsprache, und dies auch bei Vergleichsgruppen mit kontrollierter Intelligenz (Müller, 1996a; 1997). Es scheint, dass diejenigen ausländischen Schülerinnen und Schüler, welche die

\section{Tab. 4: Übertritt von vier verschiedenen Schultypen (9./10. Schuljahr) in die} Ausbildungsstränge der Sekundarstufe II (Berufsbildung und Mittelschule) und Nicht-Sekundarstufe II (Zwischenlösungen, Pendente usf.) Schuljahr 1997/98.

\begin{tabular}{|c|c|c|c|c|c|c|c|}
\hline \multirow[b]{2}{*}{ Übertritt aus: } & \multirow[t]{2}{*}{ Herkunft } & \multicolumn{3}{|c|}{ Sekundarstufe II } & \multirow{2}{*}{\multicolumn{2}{|c|}{$\begin{array}{c}\begin{array}{c}\text { Nicht } \\
\text { Sekundar- } \\
\text { stufe II }\end{array} \\
\begin{array}{c}\text { Total nicht } \\
\text { SEK II }\end{array}\end{array}$}} & \multirow[t]{2}{*}{ Total } \\
\hline & & $\begin{array}{l}\text { Berufs- } \\
\text { lehren }\end{array}$ & $\begin{array}{l}\text { Mittel- } \\
\text { schulen }\end{array}$ & $\begin{array}{c}\text { Total } \\
\text { SEK II }\end{array}$ & & & \\
\hline \multirow{2}{*}{$\begin{array}{l}\text { 3. Sekundarklasse } \\
\text { (höchste Ansprüche) }\end{array}$} & Schweiz & $613 \%$ & $18.1 \%$ & $79.4 \%$ & & $20.6 \%$ & $100 \%$ \\
\hline & Ausland & $76.7 \%$ & $11.9 \%-$ & $68.6 \%$ & & $31.4 \%$ & $100 \%$ \\
\hline \multirow{2}{*}{$\begin{array}{l}\text { 3. Realklasse } \\
\text { (mittlere Ansprüche) }\end{array}$} & Schweiz & $65.4 \%$ & - & $65.4 \%$ & \multirow{2}{*}{2} & $34.6 \%$ & $100 \%$ \\
\hline & Ausland & $46.9 \%$ & - & $46.9 \%$ & & $53.1 \%$ & $100 \%$ \\
\hline \multirow{2}{*}{$\begin{array}{l}\text { 3. Oberschule } \\
\text { (Grundansprüche) }\end{array}$} & Schweiz & $66.7 \%$ & & $66.7 \%$ & & $33.3 \%$ & $100 \%$ \\
\hline & Ausland & $35.1 \%$ & 1 & $35.1 \%$ & & $64.9 \%$ & $100 \%$ \\
\hline \multirow{2}{*}{$\begin{array}{l}\text { Zwischenlösung } \\
\text { (10. Schuljahr) }\end{array}$} & Schweiz & $74.0 \%$ & $\begin{array}{ll}- \\
-\end{array}$ & $74.0 \%$ & & $26.0 \%$ & $100 \%$ \\
\hline & Ausland & $47.3 \%$ & - & $47.3 \%$ & 3 & $52.7 \%$ & $100 \%$ \\
\hline \multirow[t]{2}{*}{ Alle Schultypen } & Schwelz & $64.5 \%$ & $6.7 \%$ & $71.2 \%$ & & $28.8 \%$ & $100 \%$ \\
\hline & Austand & $47.6 \%$ & $1.9 \%$ & $49.5 \%$ & & $50.5 \%$ & $100 \%$ \\
\hline
\end{tabular}

(Ausschnitte aus der Bildungsstatistik der Bildungsdirektion des Kantons Zürich. Persönliche Datenübermittlung des BiDZ. 1999.) 
doppelte Hürde der sozialen Schicht und der ethnolinguistischen Erschwernisse genommen hatten und dann in die Sekundarschule gelangten, dies aufgrund ihrer überdurchschnittlichen Intelligenz taten $(\mathrm{p}=0.03)$. Man kann also davon ausgehen, dass es sich bei der Gruppe der ausländischen Schülerinnen und Schüler auf ihren SEK I-Typus bezogen durchschnittlich nicht um intellektuell leistungsschwächere Schülerinnen und Schüler handelt. Heissen gleiche schulische Voraussetzungen somit gleiche Ausbildungschancen in der SEK II?

1 Tab. 4 wurde aufgrund der Gesamtschülerinnen- und Schülerstatistik des Schuljahrs 1997/98 des Kantons Zürich erstellt. Vergleicht man die Berufsbildungsquoten von ausländischen und schweizerischen Jugendlichen mit gleichem Abschluss, so fallen diese in hohem Grade zu Ungunsten der ausländischen Schulabgänger aus. Im Kanton Zürich traten (1997/98) bedeutend weniger Ausländerinnen und Ausländer eine SEK II an, und dies bei allen Typen der SEK I. Gleicher Schulabschluss heisst keinesfalls auch gleiche Chancen beim Übertritt in die SEK II. Zudem gilt: Je niedriger das Anspruchsniveau des SEK I-Schultyps, desto schwieriger gestaltet sich der Übertritt und desto geringer sind die Bildungschancen ausländischer Schülerinnen und Schüler im Vergleich zu ihren schweizerischen Klassenkameradinnen und -kameraden

2 In der Oberschule ist die Chance für eine/n AusländerIn auf 35.1\% abgesunken, bei den $\mathrm{CH}$ ist sie immerhin noch 66.7\%. Vergleicht man die Übertrittsquote von schweizerischen Oberschülerinnen und -schülern mit derjenigen ausländischer Sekundarschülerinnen und -schüler, so sind diese annähernd gleich: CH: 66.7\%; Ausland: 69.7\%. Anders gesagt: Die Chancen eines ausländischen Sekundarschülers, nach seinem obligatorischen Schulabschluss eine Berufsbildung oder weiterführende Allgemeinbildung anzutreten, sind im Kanton Zürich etwa gleich gross wie für einen Schweizer Oberschüler.

3 Überdurchschnittlich viele AUSL weichen darum auf die «Lösung» Zwischenjahr aus (CH: 14.4\%; AUSL: 22.2\%). Aber auch dieser Weg ist für einen grösseren Teil der AUSL eine Sackgasse: Es schaffen es beim zweiten Anlauf nur 47.3\% der AUSL, jedoch 74\% der Schweizerinnen und Schweizer. Betrachtet man die Situation gesamthaft, so beträgt die Übertrittsquote aller Schulabgängerinnen und -abgänger der letzten Klasse der Sekundarstufe I bei den Schweizerinnen und Schweizern 71.2\%, bei den AUSL nur 49.5\%.

\section{Zusammenfassung}

(1) In positiver Weise kann man zur Kenntnis nehmen, dass sich über die Periode von 20 Jahren die Bildungsbeteiligung auf der Sekundarstufe II insgesamt um $13.2 \%$, bei den ausländischen Schülerinnen und Schülern um 7.7\% und bei den Schweizerinnen und Schweizern um 16\% gesteigert hat (Bezugsgruppe 16-20Jährige). Hier zeigt sich bestimmt der Bedarf der Gesellschaft und der Ökono- 
mie an mehr und zugleich besser ausgebildeten Arbeitskräften. Es ist auch unzweifelhaft der Wille der Bildungsverantwortlichen dieses Landes, seine Bewohnerinnen und Bewohner als ausgebildete Menschen in die Arbeits- und Erwachsenenwelt zu entlassen. Bedenklich stimmt nun allerdings der Umstand, dass die Diskrepanz der Bildungsbeteiligung zwischen ausländischen und schweizerischen Jugendlichen wegen der unterschiedlichen Zunahmequote nochmals angewachsen ist.

(2) Eine grosse Diskrepanz besteht auch zwischen den angestrebten Ausbildungsgängen (bzw. deren Anspruchsniveaus). Die Anteile ausländischer Schülerinnen und Schüler an den Mittelschulen haben in 20 Jahren kaum zugenommen $(+0.54 \%)$. Der dortige Zuwachs geht fast ausschliesslich auf das Konto von Schweizerinnen und Schweizern $(+8.23 \%)$, und der Jugendlichen der gleichsprachigen Anrainerstaaten ohne Italien (+12.64\%).

(3) Innerhalb der Bezugsgruppe der SEK-II-AbsolventInnen (=100\%) präsentieren sich die Ergebnisse als problematisch. Hier sinken nämlich die Bildungsbeteiligungsindizes der fremdsprachigen Ausländerinnen und Ausländer in den Mittelschulen und in den Berufslehren im Verlaufe der 20 Jahre ab. Zugenommen haben bei den AUSL dafür die Vor- und Anlehren und die Zwischenlösungen, welche eher als Verlegenheitslösungen zu bezeichnen sind.

(4) Es besteht eine hierarchische Abfolge bezüglich des quantitativen Aspekts der Bildungsbeteiligung und des (qualitativen) Anspruchsniveaus gewählter Ausbildungsgänge entlang der ethnolinguistischen Linie: (1) Jugendliche aus der Schweiz und aus dem gleichsprachigen Ausland (ohne Italien) haben höheren Bildungserfolg als (2) Jugendliche aus EU-Ländern lateinischer Sprachen (ohne Frankreich) und dies wiederum als jene (3) Jugendliche aus Ex-Jugoslawien, der Türkei und Albanien. Die ethnolinguistische Herkunft erweist sich zudem als bedeutsamerer Faktor als jener von Gender, wo auf der SEK II die Werte bezüglich des Anspruchsniveaus in allen analysierten Nationen zugunsten der Frauen ausfallen.

(6) Die Bevorteilung der monolingualen Jugendlichen weist auf zwei bedenkenswerte Aspekte hin: (a) Bezüglich der Mittelschulen kann von einer eigentlichen sprachlich-nationalen Abschottung dieser Institutionen gegenüber fremdsprachigen Schülerinnen und Schüler gesprochen werden. Die Indizes in der Abbildung 2 weisen nach, dass (besonders auch in Rezessionsphasen) die monolinguale Gruppe vermehrt den am tertiären Bildungsbereich orientierten Mittelschulen zustreben, während die fremdsprachigen ausländischen Jugendlichen von diesem Trend vergleichsweise wenig profitieren können. Die zunehmende Bildungsbeteiligung ist bei dieser Gruppe fast ausschliesslich den Berufslehren, Anlehren und Zwischenjahr-Lösungen zu verdanken. Es stellt sich somit die Frage, ob die Mittelschulen ihre Integrationsaufgabe wahrnehmen oder ob sie nicht eher eine Art «gymnasiales» Ideal anstreben, das aber von der multikulturellen Wirklichkeit der ausländischen Jugendlichen weit abgehoben ist (ausführliche Diskussion in: Müller 2001, S. 42f.). (b) Man kann von einer Art Präferentialismus des schweizerischen Bildungssystems sprechen, welches (statistisch 
betrachtet) auch bei gleichen Ausbildungsvoraussetzungen dem monolingualen Jugendlichen den Vorzug gibt. Ein Teil der ausländischen Jugendlichen würde demzufolge als Reservoir für all jene leer gewordenen Ausbildungsplätze dienen, die von schweizerischen Jugendlichen nicht (mehr) beansprucht werden und die somit nicht anders besetzt werden können. Die vorliegenden Daten - insbesondere die Analyse des Bildungsbeteiligungsindizes in Abb. 2 - bestätigen diese Hypothese.

\section{Berufliches Anspruchsniveau (BAN) bei den von Schweizerinnen und Schweizern sowie Ausländer- innen und Ausländern gewählten Berufslehren: Kompensations- oder Inversionseffekt.}

Mit den eben erwähnten Berufslehren wenden wir uns abschliessend einem Bereich zu, in dem das Problem der Benachteiligung ausländischer Jugendlicher weiterhin gegeben, aber - vorsichtig gesagt - etwas weniger virulent ist: den Berufslehren. Die Daten berücksichtigen also nur jene Jugendlichen, die tatsächlich in den Berufslehren sind. Jene 16- bis 20-Jährigen, die vergeblich eine Berufslehre angestrebt haben, können hier nicht erfasst werden!

(1) Fragestellung / Hypothese: Es stellen sich folgende Fragen: (a) Wie verhält sich die Verteilung der Berufslehren auf Schweizerinnen und Schweizer und Ausländerinnen und Ausländer (bzw. auf die verschieden Nationen), wenn als Kriterium das Anspruchsniveau der verschiedenen Berufe gewählt wird? Und gibt es hier geschlechtsspezifische bzw. sozioökonomische Unterschiede? (b) Lässt sich eine Art Kompensation oder gar Inversion bezüglich der gewählten Berufslehren ausmachen, die dadurch bedingt ist, dass gut begabte ausländische Schülerinnen und Schüler, denen in überdurchschnittlichen Masse der Zugang zu den Mittelschulen verwehrt wurde, sich dafür bei der Lehrstellenwahl Berufe mit einem höherem Anspruchsniveau sichern (Kompensationshypothese) (Müller, 1998)?

(2) Definition des BAN: Unter dem beruflichen Anspruchsniveau (BAN) verstehe ich die Höhe der intellektuellen schulischen Erfordernisse, die mit dem Erlernen und Ausüben eines Berufes verbunden sind. In der vorliegenden Untersuchung wurde deshalb die Höhe des Anspruchsniveau von Berufen wie folgt festgelegt: (1) Wir wählten zunächst 95 Berufe, für die ein umfangreich validiertes Rating durch 9 Berufsberaterinnen und -berater bezüglich der intellektuellen Anforderung bereits bestand (Schallberger, 1979, 1982). (2) Diese 95 Berufe ergänzt durch weitere 35 Berufe, welche von 129 Schülerinnen und Schüler des 8., 9. und 10. Schuljahres gewählt bzw. angestrebt wurden, legten wir drei erfahrenen Berufsberaterinnen und-beratern zu einem Nachrating vor 
mit dem Ziel, etwaige Veränderung in den beruflichen Anforderungen 15 Jahre nach der ersten Einschätzung erfassen zu können und damit sicher zu gehen, dass die damaligen Einschätzungen noch zutreffen. Die Korrelation betrug $\mathrm{r}=$ $0.94^{* *}$. (3) Schliesslich wurden die Ratings der drei Berufsberaterinnen und - berater mit den durchschnittlichen Intelligenztestwerten, die Stadelmann (1990) für die 35 meist bzw. sehr oft gewählten Berufe bei $\mathrm{N}$ von 40 bis 2931 erhoben hatte, korreliert. Die Korrelation ergab ein hohes $r=0.87^{* *}$. Man kann also davon ausgehen, dass die im Folgenden dargestellten Ergebnisse bezüglich der BAN in den Lehren die Realität in hohem Grade wiedergeben. Die BAN sind hier in sechs Kategorien ( 2 bis 7 ) aufgeteilt, die in zwei Gruppen ( 2 bis $4=$ niedriges BAN; 5 bis 7 = hohes BAN) zusammengefasst werden ${ }^{8}$.

(3) Verteilung der BAN: In Abildung. 3 und Abbildung 4 sind für das Schuljahr 1998/99 die gesamtschweizerischen Daten der Verteilung der Anspruchsniveaus von 115 Berufen mit einem N von 155'064 Lehrlingen dargestellt. Vergleichen wir zunächst die beiden Säulengruppen rechts in Abbildung 3 , wo die BAN in die drei niedrigen BAN (2-4) und die drei hohen BAN (5-7) gruppiert wurden. Es zeigt sich, dass bei den oberen BAN die Schweizerinnen und Schweizer zwar mit 43\% immer noch besser als die AUSL mit 34\% abschneiden, während bei den unteren Niveaus das Verhältnis umgekehrt 66\% zu 57\% ausfällt. Jedoch haben sich die Unterschiede in den Berufslehren zugunsten der AUSL stark vermindert. Ein Blick auf die analogen Säulengruppen in Abbildung 4 zeigt, dass die niedrigeren Werte der AUSL hauptsächlich auf die Länder Türkei und Ex-Jugoslawien zurückzuführen sind. Ihre Anteile bei den drei niedrigen BAN liegen bei $70.6 \%$ bzw. 79.35\%. Bei den nach den Einzelwerten differenzierten BAN sind die Jugendlichen aus Ex-Jugoslawien und z.T. der Türkei äusserst stark im BAN 2 und BAN 3 vertreten. Nimmt man allerdings das anzahlmässig starke Italien oder die Gesamtheit der lateinisch sprechenden EU-Staaten (E/P/F/I) als Vergleich, so haben sich die Werte mit 38\% bei den höheren BAN schon sehr stark an die Schweizerwerte von $43 \%$ angenähert. Auch bei den BAN der Berufslehren bestätigt sich die bereits beschriebene Abfolge: (1) Schweizerinnen und Schweizer und Jugendliche aus Anrainerstaaten (D/A/F/FL), (2) Jugendliche aus lateinisch sprechenden EU-Staaten und (3) aus der Türkei und Ex-Jugoslawien (und übrigen Staaten), wobei die Unterschiede immerhin bedeutend geringer ausfallen.

(5) Inversion und Kompensation: Mussten wir beim Mittelschulbesuch in Tabelle 2 in Bezug auf den Erwartungswert bei den Ausländerinnen und Ausländern ein massive Unterrepräsentanz von $28.2 \%$. feststellen, so schrumpft diese bei den höheren BAN der Berufslehren auf 7.57\% zusammen, bei den Jugendlichen aus den lateinischen Ländern auf nur gar 3.67\% (verglichen mit dem mittleren Erwartungswert der Gesamtgruppe von 41.6\% für die höheren BAN) (Abb. 3 und 4). Selbst bei den Jugendlichen aus Ex-Jugoslawien, die in den Mittelschulen zu 


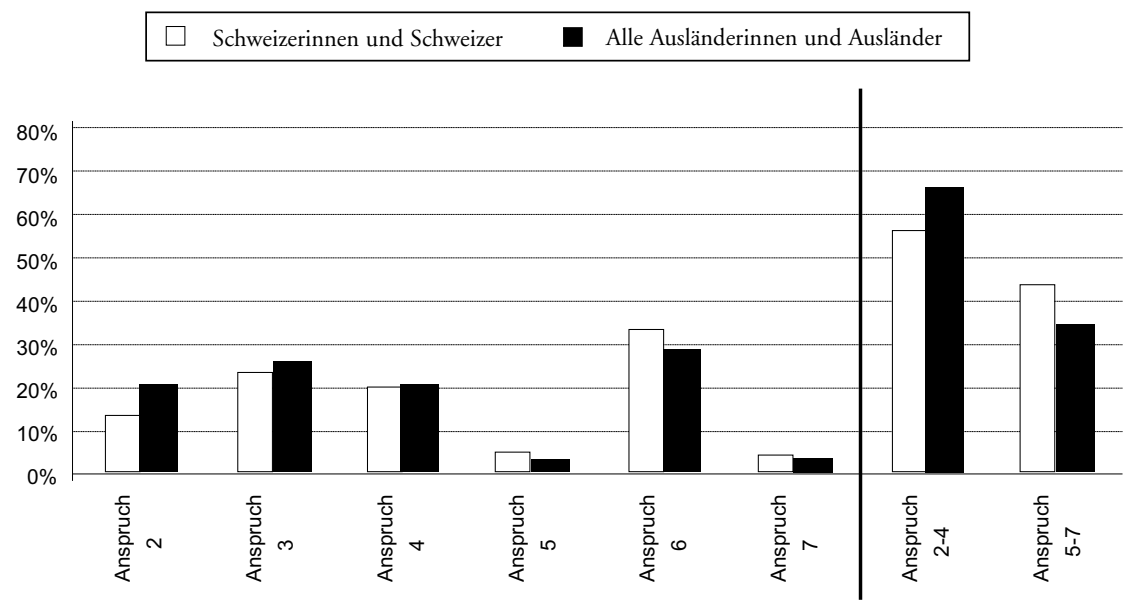

Abb. 3: Berufliche Anspruchsniveaus bei 115 Berufen (1998/99)

für SchweizerInnen (N=130'476) und AusländerInnen ( $N=24$ '588) in \%

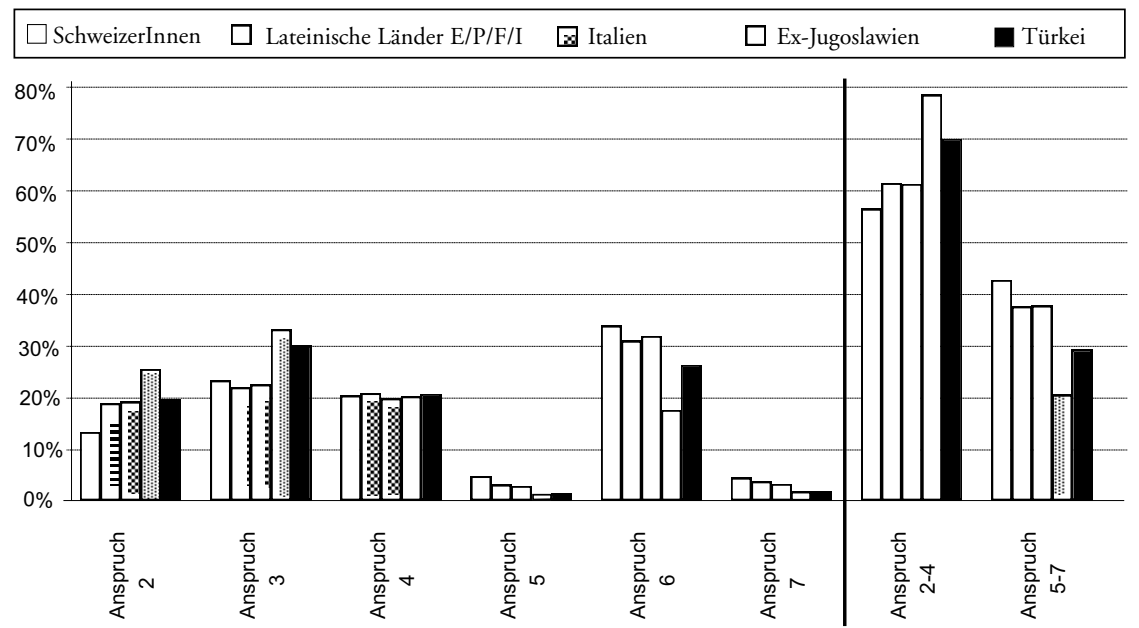

Abb. 4: Berufliche Anspruchsniveaus bei 115 Berufen (1998/99);

verschiedene Nationen 
$72.4 \%$ unterrepräsentiert sind, beträgt diese Unterrepräsentation in den BAN 57 jetzt «nur» noch 20.65\%. Es lässt sich ohne Zweifel von einer starken Verbesserung der Anteile in den anspruchsvolleren Berufslehren sprechen, wiewohl diese noch nicht als Inversion, wohl aber als Kompensation bezeichnet werden können. Es bestätigt sich somit die Hypothese, dass begabte mehrsprachige ausländische Jugendliche, die durch das schulsprachlich dominierte Selektionsverfahren von den Mittelschulen fern gehalten wurden, in Bezug auf die Berufslehren ihre Begabungen besser ins Gewicht bringen können und in einem zweiten Anlauf anspruchsvollere Lehrstellen anstreben. Zu bedenken ist auch, dass der bedeutsame Selektionsprozess am Ende der Primarstufe, also 3 bis 4 Jahre früher stattgefunden hat und dass bei später zugewanderten Schülerinnen und Schüler die schulsprachlichen Fähigkeiten wegen des nun längeren Aufenthalts in der Schweizer Schule sich vermehrt an die Norm angleichen konnten. Trotzdem stellt sich die Frage, warum es nicht zu einem vollständigen Inversionseffekt kommt. Die Gründe können hier im Einzelnen nicht diskutiert werden. Es sei aber erwähnt, dass (1) die Auslese für die Berufslehren weiterhin zu einem guten Teil auf der Beherrschung der Schulsprache beruht. So betrachten gemäss einer umfangreichen Untersuchung von Stalder (2000, S. 29) 69\% der Betriebe die Ortssprache $(\mathrm{D} / \mathrm{F})$ und den besuchten Schultypus als wichtiges Selektionskriterium, und in beiden sind die ausländischen Schülerinnen und Schüler im Nachteil. (2) Es ist wahrscheinlich, dass innerhalb des Arbeitsmarktes eine Benachteiligung oder zumindest eine Erschwerung ausländischer Arbeitnehmer und jugendlicher Stellensuchender stattfindet (Beer, 1991; König, 1991; Wimmer, 1998). (3) Die Einschätzungen von SEK I-LehrerInnen in Bezug auf das zukünftige Anspruchsniveau sind für die AUSL zu niedrig und gehen mit der Einschätzung der Deutschleistung einher, auch wenn diese Lehrerinnen und Lehrer die Mathematik und Fremdsprachenleistungen dieser gleichen Schülerinnen und Schüler höher als diejenigen der Schweizer Schülerinnen und Schüler beurteilen (Müller, 1998). Entsprechende Empfehlungen der abgebenden Lehrerinnen und Lehrer an Lehrbetriebe beeinflussen auf diese Weise die Auslese und mindern möglicherweise die eigentliche Gesamtleistung der AUSL herab.

(6) Das BAN bei Frauen und Männern: Wenden wir uns noch der Verteilung der BAN in Hinblick auf die Verteilung der Geschlechter zu (Datenbasis 24'045 Lehrlinge des Kantons Bern $=74.2 \%$ der Lehrlingsgesamtpopulation). Bei dieser Analyse muss bedacht werden, dass einerseits bestimmte Berufe typische Frauenberufe (Hotelfachassistentin, Drogistin, Kranken- und Psychiatriepflegerin) bzw. Männerberufe (z.B. Automonteur, Zimmerer, Elektromonteur, TVRadio Elektriker) sind und dass es bestimmte Berufsgruppen gibt, die (fast) ausschliesslich von Schweizerinnen und Schweizer besetzt werden (z.B. LandschaftsgärtnerIn, ZimmererIn, LandwirtIn, DrogistIn, PsychiatriepflegerIn) oder in welchen umgekehrt die AUSL überrepräsentiert sind (z.B. AutomonteurIn; HotelfachassistentIn; VerkäuferIn; BüroangestellteR; FeinmechnikerIn; TVRadio-ElektrikerIn). Bei der individuellen Berufswahl liegen somit nicht immer 
die Überlegung des BAN zugrunde. Vielmehr führen z.B. auch traditionalistische Vorstellungen oder Präferenzen auf Seiten der Betriebe dazu, einem bestimmten Geschlecht bzw. der Nationalität den Vorrang zu geben (vgl. Borkowsky, 2000). Auf diese äusserst bedeutsamen Zusammenhänge kann hier nicht eingegangen werden.
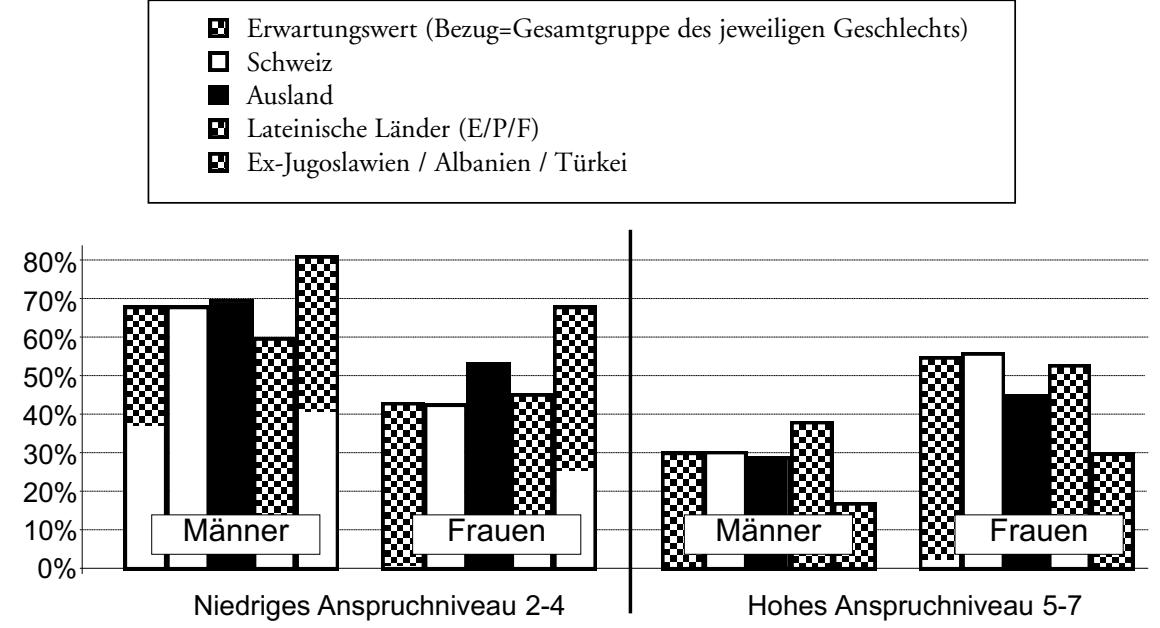

Abb. 5: Anspruchsniveau bei 115 Berufen (1998/99) in \%; Schweizer (N=12'735), Schweizerinnen (N=9'848), Ausländerer ( $N=856)$, Ausländerinnen $(N=606)$

In Abbildung 5 werden die niedrigen und die hohen BAN für vier Nationen (-gruppen) und den mittleren Erwartungswert für das jeweilige Geschlecht (BAN 5-7: MAN: 31\%; FRAU: 56\%) dargestellt. Zunächst lässt sich für diese Stichprobe eine hochgradige Annäherung der AUSL- an die CH-Lehrlinge festhalten. Sieht man einmal von den Lehrlingen aus Ex-Jugoslawien, Albanien und der Türkei ab, so sind die Werte der anzahlstarken lateinischen EU-Länder weitgehend mit denjenigen der Schweiz identisch. Wie wir schon bei den Mittelschulen festgestellt haben (Tab. 3), sichern sich auch in den Berufslehren die Frauen die intellektuell anspruchsvolleren Ausbildungsgänge. Ins Gewicht fallen hier die mit hohen Anteilen vertretenen Pflegeberufe und das starke Übergewicht der Frauen bei den kaufmännischen Angestellten (beide BAN =6). Etwas verallgemeinernd kann man sagen, dass die Frauen gegenüber den Männern sowohl in den Mittelschulen wie auch in den "höheren» Berufslehren im Verhältnis 3:2 übervertreten sind. Dies trifft auch für die ausländischen Lehrlinge zu, obwohl manchmal behauptet wird, dass die weiblichen Angehörigen aus dem islamischen Raum kulturspezifisch diskriminiert würden. Auch bei der Gruppe der Jugendlichen aus der Türkei, Ex-Jugoslawien und Albanien sind die Frauen 
im Verhältnis von 1:1.72 ähnlich übervertreten wie die Schweizerinnen (1:1.82). Insgesamt zeigt sich, dass die ethnolinguistische Zugehörigkeit sowohl bei weiblichen als auch männlichen Lehrlingen die Wahl von Berufen mit unterschiedlichem BAN steuert, und dass dadurch auch hier die ausländischen gegenüber den Schweizer Jugendlichen benachteiligt sind, wenn auch nicht mehr im gleich hohem Masse, wie an den Mittelschulen.

(6) Sozioökonomische Schichtung, Geschlechtszugehörigkeit und BAN: Die Struktur der Individualdaten des Kantons Bern ermöglicht es nicht, die Analyse nun auch noch in Richtung der sozioökonomischen Schichtung (SÖS) vorzunehmen. Eine unveröffentlichte Zweitanalyse des Datenmaterials von Müller (1993) bei 129 Schülerinnen und Schüler der 8. bis 10. Klasse gibt jedoch Hinweise auf eine höchst komplexe berufliche Wahlwirklichkeit, wenn die Analyse um den Parameter der SÖS erweitert wird. In Tabelle 5 sind die Ergebnisse im Überblick dargestellt. Die Verbindung der Parameter weiblich, ausländisch und unterschichtszugehörig wirkt sich äusserst erschwerend auf die Wahl anspruchsvollerer Berufe aus. Umgekehrt scheinen ausländische Frauen und Männer der Mittelschicht besonders vom Kompensationseffekt zu profitieren (BAN 5.5-7). Eine relativ grosse Gruppe von männlichen, ausländischen Unterschichtsangehörigen und Mittelschichtsschweizerinnen und -schweizer streben die höheren BAN zwischen 4.3 und 5.5 an. Relativ schlecht schliessen die männlichen Schweizer $\mathrm{ab}$, und zwar unabhängig davon, welcher Schicht sie angehören (BAN ( 4.3). Wahrscheinlich ist, dass es sich bei diesen Schülerinnen und Schülern statistisch um jene Gruppe handelt, bei denen - im Unterschied zur Gruppe der AUSL ein allgemeines Leistungsdefizit zum Zeitpunkt der Selektion (6. Klasse) vorhanden war, welches sie im Verlauf der SEK I nicht wettmachen konnten. Die in dieser Untersuchung gezeigten Daten legen die Vermutung nahe, dass der Kompensationseffekt sich im Falle der Berufslehren hauptsächlich bei ausländischen Mittelschichtsangehörigen, dann aber besonders bei der grossen Gruppe der männlichen Unterschichtsangehörigen positiv auswirkt. Die ausländischen Unterschichtsfrauen, die immerhin 45.9\% der AUSL-Gruppe ausmachen, bleiben aber stark benachteiligt. Jedoch befinden wir uns wegen der kleinen Stichprobe noch auf der Ebene der Hypothese, die erst durch eine repräsentative Untersuchung grösseren Umfangs geklärt werden kann.

(7) Zusammenfassung: Die Untersuchung zum beruflichen Anspruchsniveau zeigt klar auf, dass die intellektuelle Leistungsfähigkeit der ausländischen Jugendlichen diese dazu drängt, bei den Berufslehren Berufe mit höheren Anforderungen zu ergreifen, auch wenn dies nicht in der Form einer vollständigen Inversion geschieht. Wir haben behauptet, dass dieser Kompensationseffekt mit der monolingual orientierten Selektionspraxis der Schweizer Schule zu tun habe und dass die dadurch verursachte Unterrepräsentanz der AUSL in den Mittelschulen hier bis zu einem gewissen Grade wieder wettgemacht würde. Hierzu ist 
allerdings zu sagen, dass diese Behinderungen keineswegs ihr Ende gefunden haben. Auch im Übergang in die Berufslehre stellte sich heraus, dass gleicher Schulabschluss für die ausländischen Schülerinnen und Schüler nur die halbe Chance im Vergleich zu ihren Schweizer Kolleginnen und Kollegen bedeutete (Tab. 4), und dabei sind jene ausländischen Jugendlichen, die erst nach der obligatorischen Schulpflicht in die Schweiz einwanderten, noch gar nicht gerechnet. Der Kompensationseffekt ist also mitnichten jener Effekt, den die Bildungsverantwortlichen zur Überlegung verführen sollte, jetzt sei die Sache ja wieder eingerenkt. Vielmehr ist er die Auswirkung einer schulsprachlich-monolingual dominierten Selektionspraxis, die also solche nur noch die Zeit überdauert, unter dem Gesichtspunkt einer sinnvollen Auslese aber längst ausgedient hat.

Tab. 5: Verteilung der 8 SchülerInnengruppen (ethnolinguistische Zugehörigkeit (CH / AUSL) x Geschlechtszugehörigkeit (FRAU / MAN) x sozioökonomische Zugehörigkeit (SÖS 1=Untere Schicht / SÖS 2=Mittlere Schicht) auf der Skala des beruflichen Anforderungsniveaus BAN; $N=129$; Schuljahre 8, 9 und 10.

\begin{tabular}{|c|c|c|c|c|c|}
\hline BAN & niedrig & $\begin{array}{l}\text { Unteres } \\
\text { Mittel }\end{array}$ & $\begin{array}{l}\text { Oberes } \\
\text { Mittel }\end{array}$ & hoch & sehr hoch \\
\hline BAN-Wert & $\begin{array}{l}2.00 \text { bis } \\
3.89\end{array}$ & $\begin{array}{l}3.90 \text { bis } \\
4.29\end{array}$ & $\begin{array}{l}4.30 \text { bis } \\
4.69\end{array}$ & $\begin{array}{l}4.70 \text { bis } \\
5.49\end{array}$ & $\begin{array}{l}5.50 \text { bis } \\
7.00\end{array}$ \\
\hline $\begin{array}{l}\text { SÖS } 1 \\
\text { FRAU } \\
\text { (CH / AUSL) }\end{array}$ & $\begin{array}{l}\text { SÖS1 } \\
\text { FRAU } \\
\text { AUSLAND } \\
\text { BAN:3.53 } \\
\mathrm{N}: 45.9 \%\end{array}$ & $\begin{array}{l}\text { SÖS1 } \\
\text { FRAU } \\
\text { SCHWEIZ } \\
\text { BAN: } 4.00 \\
\text { N: } 20 \%\end{array}$ & & & \\
\hline $\begin{array}{l}\text { SÖS } 1 \\
\text { MAN } \\
\text { (CH / AUSL) }\end{array}$ & & $\begin{array}{l}\text { SÖS1 } \\
\text { MAN } \\
\text { SCHWEIZ } \\
\text { BAN:4.00 } \\
\text { N: } 20 \%\end{array}$ & $\begin{array}{l}\text { SÖS1 } \\
\text { MAN } \\
\text { AUSL. } \\
\text { BAN: } 4.6 \\
\text { N: } 40.5 \%\end{array}$ & & \\
\hline $\begin{array}{l}\text { SÖS } 2 \\
\text { FRAU } \\
\text { (CH / AUSL) }\end{array}$ & & & & $\begin{array}{l}\text { SÖS2 } \\
\text { FRAU } \\
\text { SCHWEIZ } \\
\text { BAN:4.8 } \\
\text { N: } 31.2 \% \\
\end{array}$ & $\begin{array}{l}\text { SÖS2 } \\
\text { FRAU } \\
\text { AUSLAND } \\
\text { BAN: } 5.50 \\
\text { N: } 5.4 \%\end{array}$ \\
\hline $\begin{array}{l}\text { SÖS } 2 \\
\text { MAN } \\
\text { (CH / AUSL) }\end{array}$ & & $\begin{array}{l}\text { SÖS2 } \\
\text { MAN } \\
\text { SCHWEIZ } \\
\text { BAN:4.00 } \\
\text { N: } 27.4\end{array}$ & & & $\begin{array}{l}\text { SÖS2 } \\
\text { MAN } \\
\text { AUSLAND } \\
\text { BAN: } 6.5 \\
\text { N: } 8.2 \%\end{array}$ \\
\hline $\begin{array}{l}\text { Berufs- } \\
\text { beispiele }\end{array}$ & $\begin{array}{l}\text { Coiffeuse } \\
\text { Malerin } \\
\text { Schneiderin }\end{array}$ & $\begin{array}{l}\text { Büroange- } \\
\text { stellte } \\
\text { Automech. } \\
\text { Verkäuferin }\end{array}$ & $\begin{array}{l}\text { Typografin } \\
\text { Feinmecha- } \\
\text { niker } \\
\text { Elektrikerin }\end{array}$ & $\begin{array}{l}\text { Grafikerin } \\
\text { EDV-Operat. } \\
\text { Schalterbe- } \\
\text { amte }\end{array}$ & $\begin{array}{l}\text { Kauf. An- } \\
\text { gestellteR } \\
\text { Bauzeich } \\
\text { nerIn } \\
\text { Elektroni- } \\
\text { kerIn }\end{array}$ \\
\hline
\end{tabular}




\section{Schluss}

Es ist hier nicht der Ort, Ratschläge an die Bildungsverantwortlichen in Hinblick auf die Verbesserung der Situation auf der SEK II zu geben. Dazu haben sich diese kürzlich selbst ein umfassendes Dossier durch Expertinnen und Experten erstellen lassen (EDK, 2000; Müller 2001, S. 47ff.). Wie so oft bei dieser föderalistischen Organisation bleibt die Befürchtung bestehen, dass das von den Ärzten verschriebene Remedium in den eigenen vier Wänden nicht angewandt wird. Es liegt meiner Auffassung nicht an einem Mangel an Kenntnissen von Sachverhalten, denn der Missstand bezüglich der Integration der ausländischen Jugendlichen liegt auf der Hand, so dass er nur noch totgeschwiegen, nicht aber mehr geleugnet werden kann. Es liegt am Ausbleiben des Handelns.

Wenn wir als objektives Mass für das Integrationspotential bzw. den Integrationswillen, wie zu Beginn dieses Artikels beschrieben, den Grad des Bildungserfolgs von ausländischen Schülerinnen und Schülern anerkennen, so liegt die Wirklichkeit noch sehr weit hinter den verbalen Verlautbarungen der Bildungsbehörden zurück. Wir müssen die massive Unterrepräsentanz von Ausländerinnen und Ausländern auf der SEK II zur Kenntnis nehmen - und dies nicht etwa nur bei jenen, die erst zugewandert sind, sondern auch bei jener sehr grossen Gruppe von Jugendlichen, die dieses Land als eines ihrer Heimatländer und diese Sprache als eine ihrer Muttersprachen bezeichnen. Wir müssen zur Kenntnis nehmen, dass selbst bei gleichen schulischen Voraussetzungen der Zugang zur SEK II durch den einfachen Umstand, nicht monolinguale Ausländerin oder Ausländer zu sein, erschwert ist. Wir müssen zur Kenntnis nehmen, dass je höher bezüglich dem Bildungsanspruch eines Schultypus ist, desto mehr er sich gegenüber den "fremdsprachigen» Ausländerinnen und Ausländern abschottet. Im Verlauf der letzten 20 Jahre wurde dieser Missstand nicht behoben, sondern im Gegenteil verschärft. Bezüglich der Mittelschulen kann man von einem eigentlichem Präferentialismus sprechen. Wie anders wäre es sonst zu erklären, dass die Anteile der Schweizerinnen und Schweizer an den Mittelschulen in dieser Periode um 8.2\% anwächst, bei den Ausländerinnen und Ausländern insgesamt nur um $0.54 \%$ ? Wir nehmen auch zur Kenntnis, dass bei den Berufslehren sich doch einiges verbessert hat und dass die durch den Abzug der Schweizerinnen und Schweizer zu den gymnasialen Bildungsgängen jetzt frei werdenden Lehrstellen vermehrt durch die Jugendlichen aus dem Ausland besetzt werden. Aber selbst hier konnten die Schweizerinnen und Schweizer mit 5.48\% noch mehr zulegen als die Gesamtgruppe der Ausländerinnen und Ausländer mit 4.84\%.

Wir konnten sehen, dass eine Art Kompensationseffekt bei den Ausländerinnen und Ausländern im Bereiche der anspruchsvolleren Berufslehren auftritt. Man mag dies als einen glücklichen Umstand bezeichnen. In Wirklichkeit aber drückt sich darin das nicht genutzte Bildungspotential der ausländischen Jugendlichen aus. Es ist eine nicht widerlegbare Tatsache, dass in der Schweizer Schule Selektionskriterien angewandt werden, die (1) mit der multilingualen 
Gegebenheit eines grossen Teils ihrer Schülerinnen und Schüler nichts zu tun haben und die (2) es verhindern, eine Auswahl zu betreiben, die sich an den Bedürfnissen einer modernen Gesellschaft und ihrer Arbeitswelt orientiert. Es dürfte Bildungsverantwortlichen schwer fallen, die Frage zu beantworten, weshalb die Beherrschung des majoritären Standards in jenem durch die monolinguale Schule geforderten Masse für die in der Schweiz ansässigen mehrsprachigen Jugendlichen so zentral sein sollte, wenn doch gleichzeitig immer mehr ausländische Personen in Betriebe eingeschleust werden, die weder diesen Standard noch die lokale Umgangssprache beherrschen, und trotzdem hochqualifizierte Arbeit verrichten.

In einer hier nicht dargestellten Studie (Müller, 2000, 2001) wurde errechnet, wie viele Schweizer Schülerinnen und Schüler sich allein wegen des schulischen Misserfolgs ihrer ausländischen Kameraden in den Schulen der Sekundarstufe I mit erweiterten Ansprüchen befinden. Für das Schuljahr 1998/99 sind es 5.6\% Schweizer SEK I-Schülerinnen und -schüler, welche ihren Erfolg 22.9\% der Ausländerinnen und Ausländer in den niedrigen SEK I-Stufe verdanken; und das sind immerhin 11'143 Schülerinnen und Schüler! Es gibt dies eine Vorstellung davon, wie gross der mögliche Verlust an Fähigkeitsressourcen sein könnte. Ich möchte dabei nicht behaupten, alle diese Schweizerinnen und Schweizer seien zu Unrecht in einer SEK I mit erweiterten Ansprüchen. Sicher aber ist, dass hier einem grossen Teil der ausländischen OberschülerInnen Unrecht geschieht.

Ich bin der Meinung, dass das überkommene Selektionssystem der schweizerischen Schule, welches sich in ihren Grundlagen über Jahrzehnte kaum verändert hat und weiterhin von einem normativen Monolingualismus dominiert wird, sich zwar zur Sicherung von Gruppenprivilegien eignet, aber in Hinblick auf viele ausländische Kinder und Jugendliche nicht jene Persönlichkeit hervorbringt, die aufgrund der erzieherischen Interventionen ihre Dispositionen voll entfalten könnte.

Der Theorie des Humankapitals folgend, wäre es die Aufgabe der Erziehung, den jungen Menschen gemäss ihrer intellektuellen Voraussetzungen jene Fähigkeiten zu vermitteln, die sie zu einem späteren Zeitpunkt fruchtbar in den gesellschaftlichen und ökonomischen Prozess einbringen können. Unter diesem Gesichtspunkt erscheint es wichtig, die selektiven Kriterien und Verfahren den Gegebenheiten des Arbeits- und des gesellschaftlichen Prozesses anzupassen. Offenbar ist dies der schweizerischen Gesellschaft (wie auch anderen westeuropäischen Staaten) noch nicht gelungen. Das notwendige Know-how muss nun auf anderem Wege von (z.T. wirtschaftlich schwachen) Ländern bezogen werden, von Menschen also, die weder mit der hiesigen Kultur noch mit der hiesigen Sprache vertraut sind. 


\section{Anmerkungen}

1 Kommissionsbericht der Kommission für Zuwanderung der Bundesregierung, Juli 2001.

2 Dies lässt sich auch in der "Europäischen Charta der Regional oder Minderheitssprachen» erkennen, in welcher die "Sprache von Zuwanderern"» vom rechtlichen Schutz ausgeschlossen wird (Art 1, Abs. aii)

3 Wäre dies der Fall, wäre ja der «Import» asiatischer Technologen unsinnig, oder studentische Austauschprogramme (z.B. Erasmus) wären weitgehend unmöglich.

4 Ich danke Herrn M. Graber vom Bundesamt für Statistik für seine Bereitschaft, auf alle meine vielfältigen Wünsche eingegangen zu sein. Seinem Einsatz ist es zu verdanken, dass die aktuellen Daten mir sofort nach deren Aufbearbeitung zur Auswertung überlassen werden konnten.

5 In diesem Sinne sind die Lehrstellenbeschlüsse I und II des Bundesamtes für Berufsbildung und Techniologie BBT zu interpretieren. Gleichzeitig besteht von Seiten der Gewerkschaftsjugend eine Initiativeingabe, die das Recht auf eine nachobligatorische Ausbildung für alle erheblich erklären will. Zudem besteht ein ausführlicher Expertenbericht der EDK (2000), der in diese Richtung zielt.

6 Mit Zwischenlösungen werden alle jene Aktivitäten bezeichnet, die sich zwischen der obligatorischen Schulzeit und der nicht obligatorischen Sekundarstufe II (z.B. Berufslehre) ansiedeln. Es sind das zur Hauptsache: 10. Schuljahr, Schnupperlehrjahre, Fremdsprachenaufenthalt, schulfreies Zwischenjahr.

7 Diese Dreigliederung der Sekundarstufe I gilt für den Kanton Zürich. Ähnliche Gliederungen haben die Kantone Aargau und Solothurn. Der Kanton Bern hat demgegenüber eine Doppelgliederung.

8 Beispiele für die jeweils häufigsten Berufe mit BAN: BAN 2: HotelfachassistentIn, BäckerIn, LandschaftsgärtnerIn, MalerIn, Coiffeur/se; AutomonteurIn (71.2\%); BAN 3: VerkäuferIn; KöchIn; LandwirtIn; ZimmererIn; MetallbauerIn, MaurerIn (66.8\%); BAN 4: ElektromonteurIn, SchreinerIn, AutomechanikerIn, MaschinmechanikerIn, MechanikerIn (76\%); BAN 5: DrogistIn, TV-Radio EletrikerIn, AugenoptikeIn, ElektromechanikerIn, GrafikerIn (83.3\%9); BAN 6: Kaufm. AngestellteR, Krankenpflege I/II, (Hoch-) BauzeichnerIn (87.7\%), BAN 7: ElektronikerIn, InformatikerIn, PsychiatriepflegerIn (100\%).

\section{Bibliographie}

Alsalam, N. \& Conley, R. (1995). The Rate of Return to Education: A Proposal for an Indicator. In OECD (Ed.), Education and employment (pp. 83-109). Paris: OECD.

Beer, D. (1991). Erfahrungen bei der Ausbildungsplatzsuche und mit den Beratungsdiensten der Bundesanstalt für Arbeit aus der Sicht ausländischer Jugendlicher. In W. Nieke \& U. Boos-Nuenning (Hrsg.), Ausländische Jugendliche in der Berufsausbildung: Auf dem Weg zur Chancengleichheit? (S. 53-62). Opladen: Leske \& Budrich..

BfS [Bundesamt für Statistik] (Hrsg.) (1996). Szenarien zur Bevölkerungsentwicklung der Schweiz. 1995-2050. Neuchâtel: BfS.

BfS (Hrsg.) (1998). Indikatoren zum Humankapital. Neuchâtel: BfS.

BfS (Hrsg.) (1999). Bildungsindikatoren Schweiz 1999. Neuchâtel: BfS.

Bolzmann, C. \& Eckmann, M. (1997). Jeunes sans diplôme: situations et perspectives. Panorama, (5), 97-98.

Borkowsky, A. (1996). Berufsbildung für Jugendliche ausländischer Staatsangehörigkeit. Interdialogos, (1), 2-6.

Borkowsky, A. (2000). Frauen und Männer in der Berufsbildung der Schweiz. Schweizerische Zeitschrift für Bildungswissenschaften, 22, (2), 279-294. 
Bundesamt für Industrie, Gewerbe und Arbeit (BIGA) Institut für Markt-und Sozialforschung (LINK) (1997). Lehrstellenbarometer. April 1997 (Ergebnisbericht zur Umfrage bei Jugendlichen und Betrieben). Bern: BIGA/OFIAMT.

Doudin, P.-A. (1998). Scolarisation des enfants portugais en Suisse. Rapport d'experts. (=Dossier 55) Berne: Conférence suisse des directeurs cantonaux de l'instruction publique.

Eckmann-Saillant, M. \& Bolzmann, C. (1994). Jeunes sans qualifications: trajectoires, situations, stratégies. Genève: Editions IES.

EDK (Schweizerische Konferenz der kantonalen Erziehungsdirektoren) (2000). Ausbildung und Integration von fremdsprachigen Jugendlichen auf der Sekundarstufe II. Expertenbericht (=Dossier 59). Bern: EDK.

Erziehungsdirektion Kt. Bern (1993). Grundsätze und Richtlinien für die Integration fremdsprachiger Kinder und Jugendlicher im Kanton Bern. Bern: Erziehungsdirektion.

Giles, H. (Ed). (1977). Language, Ethnicity and Intergroup Relations. London: Academic Press.

Giles, H., Bourhis, R. Y. \& Taylor, D. M. (1977). Toward a Theory of Language and Ethnic Group Relations. In H. Giles (Ed.), Language, Ethnicity and Intergroup Relations. London: Academic Press.

Giles, H. \& Byrne, J. L. (1982). An Intergroup Approach to Second Language Acquisition. Journal of Multilingual and Multicultural Development, 1, 17-40.

Gogolin, I. (1994). Der monolinguale Habitus der multilingualen Schule. Münster, New York: Waxmann.

Grin, F. (1994). L'économie de l'éducation et l'évaluation des systèmes de formation (PNR No. 33). Berne: Fonds national de la recherche scientifique.

Grossenbacher, S. (2000). Frauen in der beruflichen Aus- und Weiterbildung. Konsequenzen für die Geschlechterfrage in der Berufsbildung. Schweizerische Zeitschrift für Bildungswissenschaften, 22, (2), 295-311.

Hutmacher, W. (1995). Enfant d'ouvriers ou enfants d'immigrés? In E. Poglia, A.-N. PerretClermont, A. Gretler \& P. Dasen (Ed.), Pluralité culturelle et éducation en Suisse. Etre migrant II (pp. 107-136). Berne: Peter Lang.

Klingst, M. (2000). Die neuen Deutschen. Die Zeit, Wochenzeitung für Politik, Wirtschaft, Wissen und Kultur, 10.

König, P. (1991). Bereitschaft von Betrieben, ausländische Jugendliche auszubilden und einzustellen. In W. Nieke \& U. Boos-Nuenning (Hrsg.), Ausländische Jugendliche in der Berufsausbildung: Auf dem Weg zur Chancengleichheit? (S. 63-84). Opladen: Leske \& Budrich.

Kronig, W. (1996). Besorgniserregende Entwicklungen in der schulischen Zuweisungspraxis bei ausländischen Kindern mit Lernschwierigkeiten. Vierteljahrschrift für Heilpädagogik und ihre Nachbargebiete (VHN), (1), 62-79.

Kronig, W. (1997). Ist der hohe Anteil ausländischer Kinder in Kleinklassen erklärbar? Schweizerische Zeitschrift für Heilpädagogik, (6), 6-9.

Kronig, W., Haeberlin, U. \& Eckhart M. (2000). Immigrantenkinder und schulische Selektion. Pädagogische Visionen, theoretische Erklärungen und empirische Untersuchungen zur Wirkung integrierender und separierender Schulformen in den Grundschuljahren. Bern: Haupt.

Lischer, R. (1997). Integration - (k)eine Erfolgsgeschichte. Ausländische Kinder und Jugendliche im schweizerischen Bildungssystem (Publikation Nr. 15 Bildung und Wissenschaft). Bern: Bundesamt für Statistik (BfS).

Morice, A. (2000). Globalisierte Ökonomie - Arbeit ohne Grenzen. Rentable Immigranten. Le Monde Diplomatique. Schweizer Ausgabe, 20. November 2000, 6, (11) 14.

Moser, U. \& Rhyn, H. (1996). Evaluation der Sekundarstufe I im Kanton Zürich. Schulsystemvergleich. Erster Bericht. Zürich: Bildungsdirektion des Kantons Zürich.

Moser, U. \& Rhyn, H. (1997). Evaluation der Sekundarstufe I im Kanton Zürich. Bedingungen des Lernerfolgs. Zweiter Bericht. Zürich: Bildungsdirektion des Kantons Zürich. 
Moser, U. \& Rhyn, H. (1999). Evaluation der Schulqualität in den 6. Klassen des Kantons Zürich. Interner Arbeitsbericht Nr. 5 der Bildungsdirektion des Kantons Zürich. Zürich: Bildungsdirektion.

Müller, R. (1993). Sozialpsychologische Grundlagen des schulischen Zweitspracherwerbs. Eine empirische Studie bei zweisprachigen und einsprachigen Schülerinnen und Schüler aus der 6. 10. Klasse in der Schweiz (Inauguraldissertation an der phil-hist. Fakultät der Uni Bern). Bern: Psychologisches Institut.

Müller, R. (1996a). Sozialpsychologische Variablen des schulischen Zweitspracherwerbs von Migrantenkindern. In J. Hollenweger \& H. Schneider (Hrsg.), Mehrsprachigkeit und Fremdsprachigkeit in der Schweiz. Arbeit für die Sonderpädagogik? (33-89). Luzern: Edition SZH.

Müller, R. (1996b). Ist das Schulversagen von zweisprachigen Migrantenkindern selbstverständlich? Oder: Mit schulischer Auslese und Zuweisung zu Kleinklassen wird ein Missstand aus den Augen, aber nicht aus der Welt geschafft. Zeitschrift des Vereins Berner Logopäden VBL-Bulletin, (1), 3-18.

Müller, R. (1997). Sozialpsychologische Grundlagen des schulischen Zweitspracherwerbs bei zweisprachigen Migrantenschülerinnen und -schüler. Theoretische und empirische Studien bei zweisprachigen und einsprachigen SchülerInnen aus der 6.-10. Klasse in der Schweiz. (=Sprachenlandschat, Bd. 21). Aarau, Frankfurt a. M., Salzburg: Sauerländer.

Müller, R. (1998). Berufliches Wahlverhalten, berufliches Anspruchsniveaus und Bildungskarrieren von zweisprachigen Jugendlichen. Vorauswertung der Ergebnisse aus den Berufswahldaten von Schülerinnen und Schüler der 8.-10. Klasse (Unveröffentlichter Forschungsbericht des Autors). Bern: Staatliches Seminar Hofwil.

Müller, R. (2000). Über den Monolingualismus der Schweizer Schulen und seine Folgen für mehrsprachige Schülerinnen und Schüler. Babylonia, (1), 72-78.

Müller, R. (2001). Die ausländischen Jugendlichen auf der Sekundarstufe II: Probleme - Bildungsbeteiligung - Anspruchsniveau - Massnahmen. In SIBP (Hrsg.), Integration oder Reintegration? Fremdsprachige Lehrlinge und Lehrfrauen im Spannungsfeld zwischen Bleiben und Zurückkehren (S. 33-52)- (=SIBP Schriftenreihe Nr. 12). Zollikofen: Schweiz. Institut für Berufspädagogik.

Neuenschwander, M.P. (1997). Lehrvertragsauflösungen und ihre Folgen. Panorama, (4), 27 - 28.

OECD (1998). Linvestissement dans le capital humain - une comparaison internationale. Paris: OECD.

Psacharopoulos, G. (1987). Economics of Education; Research and Studies (The Cost-BenefitModel, pp. 342-347). Oxford: Pergamon Press.

Psacharopoulos, G. (1994) Returns on Investment in Education - A Global Update. World Development, 22, (9), 1325-1343.

Rüesch, P. (1998). Spielt die Schule eine Rolle? Schulische Bedingungen ungleicher Bildungschancen von Immigrantenkindern - einen Mehrebenenanalyse. Bern: Peter Lang.

Sassen, S. (1999). Guests and Aliens. New York: The New Press.

Sassen, S. (2000). Arbeit ohne Grenzen. Migration und Staatssouveränität. Le Monde Diplomatique. Schweizer Ausgabe, 20. November 2000, Nr. 11. Jg. 6, (11), 12-13.

Schallberger, U. (1979). Die Intelligenzentwicklung Jugendlicher in Abhängigkeit vom intellektuellen Anforderungsniveau der beruflichen Ausbildung. Schweizerische Zeitschrift für Psychologie, 38, (3), 200-208.

Schallberger, U. (1982). Das intellektuelle Anforderungsniveau von Lehrberufen im Urteil von Berufsberatern. Berufsberatung und Berufsbildung, (1), 11-18.

Stadelmann, J. (1990). Entwicklung der kognitiven Leistungsfähigkeit bei Jugendlichen im Verlauf des ersten Lehrjahrs (Inauguraldissertation am Psychologischen Institut der Universität Zürich). Zürich: Psychologisches Institut.

Stalder, B., (2000). Gesucht wird ... Rekrutierung und Selektion von Lehrlingen im Kanton Bern. Bern: Erziehungsdirektion des Kantons Bern/Amt für Bildungsforschung. 
Süss, D. \& Neuenschwander, M. (1997). Lehre - Ausstieg - Neuanfang. Lehrabbruch, Gesundheitsprobleme und deviantes Verhalten im Jugendalter (Forschungsprojekt des BfG Nr. 8046). Bern: Psychologisches Institut.

Tajfel, H. (1978). Social Categorization, Social Identity and Social Comparison. In H. Tajfel (Ed.), Differentiation between Social Groups (pp. 61-76). London: Academic Press.

Tajfel, H. (1982). Social Identity and Intergroup Relations. Cambridge: Cambridge University Press.

Tajfel, H. \& Turner, J. (1979). An Integrative Theory of Intergroup Conflict. In W. G. Austin \& St. Worchel (Ed.), The Social Psychology of Intergroup Relations (pp. 33-47). Monterey: Brooks and Cole.

Turner, J. C. (1982). Towards a Cognitive Redefinition of the Social Group. In: Tajfel, H. (Ed.), Social Identity and Intergroup Relations (pp. 15-40). Cambridge: Cambridge University Press.

Turner, J. C. \& Brown, R. (1978). Social Status, Cognitive Alternatives and Intergroup Relations. In H. Tajfel (Ed.), Differentiation between Social Groups (pp. 201-234). London: Academic Press.

Wimmer, A. (1998). Diskriminierung bei der Arbeitssuche. Skizze einer Studie zu einem unerforschten Thema. Tangram, 4, 35-38.

Wolter, S. C. \& Weber, B. A. (1998). Rates of Return to Education - Continental Europe's Dilemma. 62nd Annual Meeting of the Midwestern Economics Association (18. to 21 march 1998). Chicago.

Wolter, S.C. \& Weber, B. A. (1999a). On the Measurement of Private Raters of Return to Education. Jahrbücher f. Nationalökonomie und Statistik, 218, (5+6), 607-618.

Wolter, S.C. \& Weber, B. A. (1999b). Skilling the Unskilled - A Question of Incentives? International Journal of Manpower, 20, (3/4), 254-269.

\section{La situation des jeunes de langue étrangère au degré secondaire II du système scolaire suisse - Intégration ou discrimination?}

\section{Résumé}

Selon la théorie du capital humain, l'école devrait promouvoir de manière optimale les ressources intellectuelles de l'ensemble des élèves. Une comparaison entre jeunes autochtones suisses et certains groupes de jeunes étrangers au niveau de leur taux de scolarisation au degré secondaire II montre que celui-ci est particulièrement bas chez ces derniers. L'accès aux écoles moyennes (p. ex. gymnase), mais aussi aux apprentissages à haut degré d'exigences s'avère spécialement difficile. Cependant, un certain effet de compensation peut être observé en ce qui concerne la formation professionnelle, du fait qu'une proportion équivalente de jeunes suisses et de jeunes étrangers fréquentent des filières de formation professionnelle à exigences élevées. L'auteur critique le système de formation suisse en l'accusant de " préférentialisme ", ce dernier donnant l'avantage à l'élève monolingue même si ses performances scolaires sont plus faibles que celles de l'élève étranger bilingue. Ce " préférentialisme " servirait à protéger les intérêts directs du groupe autochtone, mais nuirait à long terme aux intérêts sociaux et économiques de l'Etat. 


\section{La situazione degli allievi stranieri a livello di secondario II: integrazione o pregiudizio?}

\section{Riassunto}

Secondo la teoria del capitale umano, la scuola dovrebbe fare tutto il possibile per promuovere in modo ottimale le risorse intellettuali degli allievi. Il confronto tra allievi di nazionalità estera e allievi svizzeri mostra però che la frequenza dei primi della scuola a livello di secondario II è bassa. E' soprattutto l'accesso alla formazione liceale a creare difficoltà, ma anche la formazione nelle professioni altamente qualificate suscita problemi. Nel caso degli apprendistati però, si nota un effetto di compensazione consistente nel fatto che una parte di stranieri comparabile agli svizzeri si ritrova nelle professioni con esigenze formative elevate. L'autore critica il sistema scolastico svizzero in quanto dà la preferenza all'allievo monolingue svizzero rispetto al plurilingue straniero. Di fatto ciò equivale ad un protezionismo in favore dei gruppi autoctoni e comporta una minaccia per gli interessi sociali ed economici a lungo termine.

\section{Foreign Students' Situation on the Secondary Level (Gymnasium; Apprenticeships) in Swiss Schools: Integrated or Disadvantaged?}

\section{Summary}

In accordance with the theory of human capital, schools must develop the educational potentials of their students. However, the comparison of several groups of foreign students with their autochthonous Swiss counterparts shows that participation of the former on the secondary II level is low. It proves to be especially difficult for foreign students to accede to the higher level education in middle schools (e.g. gymnasiums) but also in higher level apprenticeships. However, in the case of apprenticeships a compensation effect is to be noticed in the fact that a proportion of foreign students similar to that of Swiss students is to be found in higher level apprenticeships. The author blames Swiss educational systems for preferentialism, which consists in giving advantages to monolingual students, even in cases where school performances of their bilingual foreign colleagues are higher. This preferentialism helps to protect the utilitarian interests of the autochthonous group, but on a long term range it will harm the higher social and economic interests of the state. 